\title{
A SPECIAL CONFIGURATION OF 12 CONICS AND GENERALIZED KUMMER SURFACES
}

\author{
DAVID KOHEL, XAVIER ROULLEAU, ALESSANDRA SARTI
}

\begin{abstract}
A generalized Kummer surface $X$ obtained as the quotient of an abelian surface by a symplectic automorphism of order 3 contains a $9 \mathbf{A}_{2}$-configuration of $(-2)$-curves. Such a configuration plays the role of the $16 \mathbf{A}_{1}$-configurations for usual Kummer surfaces. In this paper we construct 9 other such $9 \mathbf{A}_{2}$-configurations on the generalized Kummer surface associated to the double cover of the plane branched over the sextic dual curve of a cubic curve. The new $9 \mathbf{A}_{2}$-configurations are obtained by taking the pullback of a certain configuration of 12 conics which are in special position with respect to the branch curve, plus some singular quartic curves. We then construct some automorphisms of the K3 surface sending one configuration to another. We also give various models of $X$ and of the generic fiber of its natural elliptic pencil.
\end{abstract}

[2000]Primary: 14J28

\section{INTRODUCTION}

A Kummer surface $\operatorname{Km}(A)$ is the minimal desingularization of the quotient of an abelian surface $A$ by the involution [-1]. It is a K3 surface containing 16 disjoint (-2)-curves, which lie over the 16 singularities of $A /\langle[-1]\rangle$. We call such set of curves a $16 \mathbf{A}_{1}$-configuration. A well-known result of Nikulin [17 gives the converse: if a K3 surface contains a $16 \mathbf{A}_{1}$-configuration, then it is the Kummer surface of an abelian surface $A$, such that the $16(-2)$-curves lie over the singularities of $A /\langle[-1]\rangle$.

Shioda 30] then asked the following question: if two abelian surfaces $A$ and $B$ satisfy $\operatorname{Km}(A) \simeq \operatorname{Km}(B)$, is it true that $A \simeq B$ ? Gritsenko and Hulek [13] gave a negative answer to that question in general. In [21], [22], we studied and constructed examples of two $16 \mathbf{A}_{1}$-configurations on the same Kummer surface such that their associated abelian surfaces are not isomorphic.

Kummer surfaces have natural generalizations to quotients of an abelian surface $A$ by other symplectic groups $G \subseteq \operatorname{Aut}(A)$. If $G \cong \mathbb{Z} / 3 \mathbb{Z}$, then the quotient surface $A / G$ has 9 cusp singularities, in bijection with the fixed points of $G$. Its minimal desingularization, denoted by $\mathrm{Km}_{3}(A)$, is a $\mathrm{K} 3$ surface which contains 9 disjoint $\mathbf{A}_{2}$-configurations, i.e. pairs $\left(C, C^{\prime}\right)$ of $(-2)$-curves such that $C C^{\prime}=1$. It is then natural to ask if an isomorphism

Date: May 18, 2021. 
$\mathrm{Km}_{3}(A) \simeq \mathrm{Km}_{3}(B)$ between two generalized Kummer surfaces implies that $A$ and $B$ are isomorphic.

With this question in mind, in the present paper we construct geometrically several $9 \mathbf{A}_{2}$-configurations on some generalized Kummer surfaces previously studied by Birkenhake and Lange [7]. Their construction is as follows. For $\lambda$ generic, the dual of a cubic curve $E_{\lambda}: x^{3}+y^{3}+z^{3}-3 \lambda x y z=0$ is a sextic curve $C_{\lambda}$ with a set $\mathcal{P}_{9}$ of $9 \mathbf{A}_{2}$ singularities corresponding to the nine inflection points on $E_{\lambda}$. The minimal desingularization $X_{\lambda}$ of the double cover of $\mathbb{P}^{2}$ branched over $C_{\lambda}$ is a generalized Kummer surface with a natural $9 \mathbf{A}_{2}$-configuration $\mathcal{A}_{0}$. The surface $X_{\lambda}$ has a natural elliptic fibration $\varphi: X_{\lambda} \rightarrow \mathbb{P}^{1}$ for which the $18(-2)$-curves in the $9 \mathbf{A}_{2}$-configuration are sections, and the reduced strict transform of $C_{\lambda}$ is a fiber.

In order to find other $(-2)$-curves on $X_{\lambda}$ we study the set $\mathcal{C}_{12}$ of conics that contain at least 6 points in $\mathcal{P}_{9}$. One has

Theorem 1. The set $\mathcal{C}_{12}$ has cardinality 12 . Each conic in $\mathcal{C}_{12}$ contains exactly 6 points in $\mathcal{P}_{9}$ and through each point in $\mathcal{P}_{9}$ there are 8 conics. The sets $\left(\mathcal{P}_{9}, \mathcal{C}_{12}\right)$ form therefore a $\left(9_{8}, 12_{6}\right)$-configuration.

The configuration $\left(\mathcal{P}_{9}, \mathcal{C}_{12}\right)$ has interesting symmetries, e.g. there are 8 conics among the 12 passing through a fixed point $q$ in $\mathcal{P}_{9}$ and the 8 points in $\mathcal{P}_{9} \backslash\{q\}$, which form a 85 point-conic configuration. The freeness of the arrangement of curves $\mathcal{C}_{12}$ is studied in [19, where we learned that this configuration has been also independently discovered in [11].

The irreducible components of the curves in the K3 surface $X_{\lambda}$ above the 12 conics are $24(-2)$-curves. This set of $(-2)$-curves contains nine $8 \mathbf{A}_{2}$ subconfigurations which are the strict transform of the nine $8_{5}$ sub-configurations of conics. Using the pullback to $X_{\lambda}$ of some 9 special (singular) quartic curves, we are able to complete each of these $8 \mathbf{A}_{2}$-configurations into a new $9 \mathbf{A}_{2}$-configuration $\mathcal{A}_{k},(k \in\{1, \ldots, 9\})$.

According to 4, to a $9 \mathbf{A}_{\mathbf{2}}$-configuration corresponds an Abelian surface $A$ and an order 3 symplectic group $G$ such that $X_{\lambda} \simeq \operatorname{Km}_{3}(A)$ and the $9 \mathbf{A}_{\mathbf{2}}$-configuration is the exceptional divisor of the minimal desingularisation $\operatorname{Km}_{3}(A) \rightarrow A / G$ (this is the analog of Nikulin's result for $16 \mathbf{A}_{\mathbf{1}^{-}}$ configurations).

A Kummer structure on $\mathrm{K} 3$ surface $X$ is an isomorphism class of Abelian surfaces $A$ such that $X \simeq \operatorname{Km}(A)$. Kummer structures are in one-to-one correspondence with the orbits under $\operatorname{Aut}(X)$ of Nikulin's $16 \mathbf{A}_{\mathbf{1}}$-configurations (see e.g. [21, Proposition 21]).

Similarly, one can define a generalized Kummer structure on a K3 surface $X$ as an isomorphism class of pairs $(A, G)$ of abelian surfaces $A$ and order 3 symplectic group $G$ such that $X \simeq \operatorname{Km}_{3}(A)$. We show that there is again a one-to-one correspondence between generalized Kummer structures and the orbits of the $9 \mathbf{A}_{\mathbf{2}}$-configurations. Using the $9 \mathbf{A}_{\mathbf{2}}$-configurations we constructed, we obtain: 
Theorem 2. The $9 \mathbf{A}_{\mathbf{2}}$-configurations $\mathcal{A}_{1}, \ldots, \mathcal{A}_{9}$ we obtained on the $K 3$ surface $X_{\lambda}$ are contained in the $\operatorname{Aut}\left(X_{\lambda}\right)$-orbit of $\mathcal{A}_{0}$.

For the proof we construct automorphisms sending one configuration to another. Some of these automorphisms are obtained by using translations by torsion sections of the natural elliptic fibration $\varphi: X_{\lambda} \rightarrow \mathbb{P}^{1}$, and some other by using the Torelli Theorem for K3 surfaces.

We then continue our study of the surface $X_{\lambda}$ by obtaining various models in projective space, in particular as a degree 8 non-complete intersection in $\mathbb{P}^{5}$. We construct a model of the generic fiber $E_{K 3}$ of the fibration $\varphi$.

Theorem 3. The fibration $\varphi: X_{\lambda} \rightarrow \mathbb{P}^{1}$ has 8 singular fibers of type $\tilde{\mathbf{A}}_{2}$; the $24(-2)$-curves above the 12 conics in $\mathcal{C}_{12}$ are contained in these fibers. A Hessian model of the generic fiber of $\varphi$ is

$$
E_{K 3}: x^{3}+y^{3}+z^{3}+\frac{\lambda^{3}\left(t^{2}+3\right)-4 t^{2}}{\lambda^{2}\left(t^{2}-1\right)} x y z=0 .
$$

We also get a Weierstrass model of $E_{K 3}$. It turns out that the MordellWeil group of the fibration $\varphi$ has rank 1 and torsion $(\mathbb{Z} / 3 \mathbb{Z})^{2}$; we compute its generators. Using the translation maps constructed from the model $E_{K 3}$, we can acquire other $9 \mathbf{A}_{2}$-configurations from the previously known one. We also obtain another construction of the K3 surface $X_{\lambda}$ as a double plane:

Theorem 4. The surface $X_{\lambda}$ is the minimal desingularization of the double cover of $\mathbb{P}^{2}$ branched over the sextic curve which is the union of the elliptic curves $E_{\lambda}$ and its Hessian

$$
\operatorname{He}(\lambda): x^{3}+y^{3}+z^{3}+\frac{\left(\lambda^{3}-4\right)}{\lambda^{2}} x y z=0,
$$

The strict transform on $X_{\lambda}$ of the 12 lines of the Hesse arrangement in $\mathbb{P}^{2}$ are the $24(-2)$-curves above the 12 conics.

Acknowledgements The authors wish to thank Cédric Bonnafé, Igor Dolgachev, Antonio Laface, Ulf Persson, Piotr Pokora, Giancarlo Urzúa, and also Carlos Rito for sharing his program LinSys. Part of the computations were done using Magma software [8]. The second author thanks the MaxPlanck Institute for Mathematics of Bonn for its hospitality and support. The third author is partially supported by the ANR project No. ANR-20CE40-0026-01 (SMAGP).

\section{Preliminaries}

2.1. Notations and conventions. Let $\eta: Y \rightarrow Z$ be a dominant map between two surfaces and let $C \hookrightarrow Z$ be a curve. In this paper, the reduced pullback of $C$ minus the irreducible components contracted by $\eta$ is called the strict transform of $C$ on $Y$. 
Definition 5. We say that two (-2)-curves $E, E^{\prime}$ on a $\mathrm{K} 3$ surface form an $\mathbf{A}_{2}$-configuration if their intersection matrix is: $\left(\begin{array}{cc}-2 & 1 \\ 1 & -2\end{array}\right)$. We say that the (-2)-curves $E_{1}, E_{1}^{\prime}, \ldots, E_{n}, E_{n}^{\prime}$ form an $n \mathbf{A}_{2}$-configuration if the curves $E_{j}, E_{j}^{\prime}, j \in\{1, \ldots, n\}$ form $n$ disjoint $\mathbf{A}_{2}$-configurations.

We recall (see [10]) that a $\left(v_{r}, b_{k}\right)$-configuration is the data of two sets $\mathcal{P}_{v}, \mathcal{L}_{b}$ of respective cardinality $v$ and $b$, plus a subset $I \subset \mathcal{P}_{v} \times \mathcal{L}_{b}$, such that for each element $p$ in $\mathcal{P}_{v}$, there are $r$ elements $l$ in $\mathcal{L}_{b}$ such that $(p, l) \in I$, and for each element $l$ in $\mathcal{L}_{b}$, there are $k$ elements $p$ in $\mathcal{P}_{v}$ such that $(p, l) \in I$. One has $v r=b k$. When $v=b$ and $r=k$, we call such a configuration a $v_{r}$-configuration.

2.2. Generalized Kummer structures. Let $X$ be a K3 surface. Suppose that $\mathcal{C}=A_{1}, A_{1}^{\prime}, \ldots, A_{9}, A_{9}^{\prime}$ is a $9 \mathbf{A}_{2}$-configuration on $X$. By the results of Barth [4, there exist coefficients $a_{j}, a_{j}^{\prime} \in\{1,2\}$ and a divisor $D \in \mathrm{NS}(X)$ such $\sum_{j=1}^{9}\left(a_{j} A_{j}+a_{J}^{\prime} A_{j}^{\prime}\right)=3 D$. Let $\tilde{X} \rightarrow X$ be the blow-up at the 9 intersection points $A_{k} A_{k}^{\prime}(k=1, \ldots, 9)$. The strict transform of $\mathcal{C}$ on $\tilde{X}$ is the union of 18 disjoint $(-3)$-curves. The quoted results of Barth gives that there exists a unique triple cover map $\tilde{B} \rightarrow \tilde{X}$ (for the theory of cyclic covers, see [6, Chapter 1, Section 17]) branched over the 18 (-3)-curves; the reduced pull-back of these curves are $(-1)$-curves and the pull-back of the exceptional curves on $\tilde{X}$ are $9(-3)$-curves. The minimal model of $\tilde{B}$ is then an abelian surface $B$ with an order 3 symplectic automorphism group $G$ coming from the cyclic cover, and the surface $X$ is (isomorphic to) the minimal desingularization of the quotient surface $B / G$.

Definition 6. A generalized Kummer structure (of order 3) on a K3 surface $X$ is an isomorphism class of pairs $(A, G)$ of abelian surfaces equipped with an order 3 symplectic automorphism subgroup $G \subset \operatorname{Aut}(A)$, such that $X \simeq$ $\mathrm{Km}_{3}(A)$, where $\mathrm{Km}_{3}(A)$ is the minimal desingularization of $A / G$.

Proposition 7. There is a one-to-one correspondence between Kummer structures on $X$ and $\operatorname{Aut}(X)$-orbits of $9 \mathbf{A}_{2}$-configurations.

Proof. Let $\mu: X \rightarrow X$ be an automorphism of $X$ sending the configuration $\mathcal{C}$ to the configuration $\mathcal{C}^{\prime}$. Let $B, B^{\prime}$ be the abelian surfaces and let $G \subset$ $\operatorname{Aut}(B), G^{\prime} \subset \operatorname{Aut}\left(B^{\prime}\right)$ be the order 3 automorphism groups such that $X$ is the minimal desingularization of $B / G$ and $B^{\prime} / G^{\prime}$, and the exceptional curves of the minimal desingularization are respectively in $\mathcal{C}, \mathcal{C}^{\prime}$. Let us prove that there exists an isomorphism $\tau$ between the abelian surfaces $B, B^{\prime}$ such that $G^{\prime}=\tau G \tau^{-1}$.

As above let $\tilde{X}, \tilde{X}^{\prime}$ be the blow-up of $X$ at the 9 singular points of $\mathcal{C}$ and $\mathcal{C}^{\prime}$ respectively. The automorphism $\mu$ extends to an isomorphism $\tilde{\mu}: \tilde{X} \rightarrow \tilde{X}^{\prime}$. The map $\tilde{B} \rightarrow \tilde{X} \stackrel{\tilde{\mu}}{\rightarrow} \tilde{X}^{\prime}$ is branched with order 3 over the strict transform of $\mathcal{C}^{\prime}$ in $\tilde{X}^{\prime}$. By uniqueness of the triple cover, there is an isomorphism $\tilde{\tau}: \tilde{B} \rightarrow \tilde{B}^{\prime}$, (which gives an isomorphism to $\tau: B \rightarrow B^{\prime}$ between the minimal 
models). Moreover, the order 3 automorphisms group $\tilde{G}$ of transformations of the triple cover $\tilde{B} \rightarrow \tilde{X}$ is sent by transport of structures to the order 3 automorphism group $\tilde{G}^{\prime}$ of transformations of the triple cover $\tilde{B}^{\prime} \rightarrow \tilde{X}^{\prime}$, i.e. $\tilde{G}^{\prime}=\tilde{\tau} \tilde{G} \tilde{\tau}^{-1}$. This property is preserved when taking the minimal models: $G^{\prime}=\tau G \tau^{-1}$.

For the converse, let $(B, G)$ and $\left(B^{\prime}, G^{\prime}\right)$ be equipped abelian surfaces such that $\operatorname{Km}_{3}(B)=X=\mathrm{Km}_{3}\left(B^{\prime}\right)$ and there is an isomorphism $\tau: B \rightarrow B^{\prime}$ with $G=\tau^{-1} G^{\prime} \tau$. The map $B \rightarrow B^{\prime} \rightarrow B^{\prime} / G^{\prime}$ is 3 to 1 and $G$-invariant, thus it induces an isomorphism $B / G \simeq B^{\prime} / G^{\prime}$. That isomorphism extends to the desingularization: $X=\mathrm{Km}_{3}(B) \simeq \mathrm{Km}_{3}\left(B^{\prime}\right)=X$ and sends the first $9 \mathbf{A}_{2}$-configuration to the second.

2.3. The Néron-Severi and transcendental lattices. In this Section, which can be skipped in a first reading, we compute the Néron-Severi lattice and the transcendent lattice of the generalized Kummer surface $X$, the results will be used in Section 3 ,

2.3.1. The Néron-Severi lattice. Let $A$ be an abelian surface with a symplectic action of a group $G:=\mathbb{Z} / 3 \mathbb{Z}$. The quotient $A / G$ has $9 \mathbf{A}_{2}$ singularities and the minimal resolution is a generalized Kummer surface $X:=\mathrm{Km}_{3}(A)$ which carries a $9 \mathbf{A}_{2}$-configuration $A_{1}, A_{1}^{\prime}, \ldots, A_{9}, A_{9}^{\prime}$ of $(-2)$-curves.

Observe that the abelian surface has Picard number at least 3 , see [5, Proposition on p. 10] and the K3 surface $X$ has generically Picard number $\rho(X)$ equal to 19 . Let $\mathcal{K}_{3}$ denote the minimal primitive (rank 18) sub-lattice of the $\mathrm{K} 3$ lattice $H^{2}(X, \mathbb{Z})$ containing the 9 configurations $\mathbf{A}_{2}$. The lattice $\mathcal{K}_{3}$ is described as follows. By [3, Proof of Proposition 1.3], it is generated by the classes $A_{1}, A_{1}^{\prime}, \ldots, A_{9}, A_{9}^{\prime}$ and the following three classes

$$
\begin{gathered}
v_{1}=\frac{1}{3} \sum_{i=1}^{9}\left(A_{i}-A_{i}^{\prime}\right) \\
v_{2}=\frac{1}{3}\left(\left(A_{2}-A_{2}^{\prime}\right)+2\left(A_{3}-A_{3}^{\prime}\right)+A_{6}-A_{6}^{\prime}+2\left(A_{7}-A_{7}^{\prime}\right)+A_{8}-A_{8}^{\prime}+2\left(A_{9}-A_{9}^{\prime}\right)\right) \\
v_{3}=\frac{1}{3}\left(A_{4}-A_{4}^{\prime}+2\left(A_{5}-A_{5}^{\prime}\right)+A_{6}-A_{6}^{\prime}+2\left(A_{7}-A_{7}^{\prime}\right)+2\left(A_{8}-A_{8}^{\prime}\right)+A_{9}-A_{9}^{\prime}\right) .
\end{gathered}
$$

Then the discriminant group $\mathcal{K}_{3}^{\vee} / \mathcal{K}_{3}$ is generated by the classes

$$
\begin{gathered}
w_{1}=\frac{1}{3}\left(A_{5}-A_{5}^{\prime}+A_{7}-A_{7}^{\prime}+A_{8}-A_{8}^{\prime}\right) \\
w_{2}=\frac{1}{3}\left(2\left(A_{4}-A_{4}^{\prime}\right)+A_{6}-A_{6}^{\prime}+2\left(A_{7}-A_{7}^{\prime}\right)+A_{8}-A_{8}^{\prime}\right) \\
w_{3}=\frac{1}{3}\left(A_{3}-A_{3}^{\prime}+A_{5}-A_{5}^{\prime}+A_{6}-A_{6}^{\prime}\right)
\end{gathered}
$$

with intersection matrix (the coefficients are in $\mathbb{Q} / 2 \mathbb{Z}$ ):

$$
\left(\begin{array}{ccc}
0 & 0 & -\frac{2}{3} \\
0 & -\frac{2}{3} & -\frac{2}{3} \\
-\frac{2}{3} & -\frac{2}{3} & 0
\end{array}\right) .
$$

Assume $\rho(X)=19$ (the minimal possible) and that $D_{2}$, a generator of $\mathcal{K}_{3}^{\perp} \subset \operatorname{NS}(X)$, has square $D_{2}^{2}=2$. We state here the following Lemma for later use in Sections 4.15 .3 .

Lemma 8. The Néron-Severi lattice is $\mathrm{NS}(X)=\mathbb{Z} D_{2} \oplus \mathcal{K}_{3}$ and its discriminant group has order 54 . 
Proof. The lattice $\mathcal{K}_{3}$ is 3 -elementary and $D_{2}^{2}=2$, thus the result.

2.3.2. The transcendental lattice. Since $\mathrm{NS}(X)=\mathbb{Z} D_{2} \oplus \mathcal{K}_{3}$, we know that the discriminant group is

$$
\frac{\mathrm{NS}(X)^{\vee}}{\operatorname{NS}(X)}=\frac{\left(\mathbb{Z} D_{2}\right)^{\vee}}{\mathbb{Z} D_{2}} \oplus \frac{\mathcal{K}_{3}^{\vee}}{\mathcal{K}_{3}} .
$$

The intersection matrix of the generators $w_{1}, w_{2}, w_{3}-w_{1}-w_{2}+\frac{1}{2} D_{2}$ is

$$
\mathcal{N}:=\left(\begin{array}{ccc}
0 & 0 & -\frac{2}{3} \\
0 & -\frac{2}{3} & 0 \\
-\frac{2}{3} & 0 & \frac{1}{2}
\end{array}\right) .
$$

Recall that the quadratic form on the discriminant group has values in $\mathbb{Q} / 2 \mathbb{Z}$ and the corresponding bilinear form has values in $\mathbb{Q} / \mathbb{Z}$, see e.g. [16, Section 2]. The transcendental lattice $T_{X}$ of $X$ has rank 3 signature $(2,1)$, and since $H^{2}(X, \mathbb{Z})$ is unimodular $\operatorname{det} T_{X}=-2 \cdot 3^{3}$ with discriminant form the same as the discriminant form of $\mathrm{NS}(X)$ scaled by -1 . As in [23, Definition 2.1], we define

Definition 9. We call the discriminant $d$ of an indefinite rank 3 lattice small if $4 \cdot d$ is not divisible by $k^{3}$ for any non square natural number $k$ congruent to 0 or 1 modulo 4 .

The lattice $T_{X}$ is small, thus by [9, Theorem 21, p. 395] and [9, Corollary 1.9.4] it is uniquely determined by its signature and its discriminant form. Consider now the rank three lattice $T$ of signature $(2,1)$ with Gram matrix

$$
\left(\begin{array}{ccc}
0 & 3 & 0 \\
3 & 6 & -3 \\
0 & -3 & 6
\end{array}\right)
$$

It has determinant $-2 \cdot 3^{3}$ and if one calls $u_{1}, u_{2}, u_{3}$ its generators, one computes that the discriminant group is generated by

$$
\frac{2 u_{1}}{3}, \frac{u_{3}}{3}, \frac{3 u_{1}+2 u_{2}+u_{3}}{6}
$$

and the discriminant form is the same as $-\mathcal{N}$ so that by unicity:

Proposition 10. The transcendental lattice $T_{X}$ is isometric to $T$.

2.4. The Hesse, dual Hesse, and related configurations. Let us recall the construction and properties of the Hesse configuration before introducing a configuration with analogous properties in the next section.

Let $E \hookrightarrow \mathbb{P}^{2}$ be a smooth cubic curve and $\mathcal{T}_{9}$ its set of 9 inflection points. Fixing an inflection point as the group identity, the set $\mathcal{T}_{9}$ is the 3 -torsion subgroup $E[3]$. The Hesse configuration is defined to be the pair $\left(\mathcal{T}_{9}, \mathcal{L}_{12}\right)$, where $\mathcal{L}_{12}$ is the set of 12 lines through pairs of points in $\mathcal{T}_{9}$. Since each line meets $\mathcal{T}_{9}$ at 3 points and each point of $\mathcal{T}_{9}$ is contained in 4 lines, the Hesse configuration is a $\left(9_{4}, 12_{3}\right)$-configuration. Removing one point of the 
Hesse configuration and its 4 incident lines, one obtains a symmetric $8_{3}$ configuration of 8 points and 8 lines.

We note that the construction is not unique to the curve $E$. If $\mathcal{P}$ is the set of inflection points of any nonsingular cubic plane curve $E$, then there exists a pencil of elliptic curves (called the Hesse pencil) whose set of inflection points is $\mathcal{P}$.

By taking the 12 points and 9 lines in the dual space $\left(\mathbb{P}^{2}\right)^{*}$ corresponding to the 12 lines and 9 points dual to the Hesse configuration, one obtains a dual $\left(122_{3}, 9_{4}\right)$-configuration called the dual Hesse configuration. As above, removing one line and the 4 points it meets gives a dual symmetric $8_{3}$ configuration.

As abstract configurations, the above configurations can be realized from the symmetric $134_{4}$-configuration of points and lines in the plane $\mathbb{P}^{2}\left(\mathbb{F}_{3}\right)$. Removing a fixed point and the set of 4 lines which pass through it, gives the $\left(12_{3}, 9_{4}\right)$-configuration, and removing a fixed line and the 4 points it contains gives the $\left(9_{4}, 12_{3}\right)$-configuration. The $\left(9_{4}, 12_{3}\right)$-configuration is naturally identified with $\mathbb{A}^{2}\left(\mathbb{F}_{3}\right)=\mathbb{P}^{2}\left(\mathbb{F}_{3}\right) \backslash \mathbb{P}^{1}\left(\mathbb{F}_{3}\right)$ equipped with its system of affine lines, consistent with the identification $\mathcal{T}_{9}=E[3] \cong \mathbb{F}_{3}^{2}$ in the Hesse configuration.

\section{Nine NEW $9 \mathbf{A}_{2}$-CONFigurations}

3.1. $\left(9_{8}, 12_{6}\right)$ and $8_{5}$-configurations of conics. Let us fix $\lambda \notin\left\{1, \omega, \omega^{2}\right\}$, for $\omega$ such that $\omega^{2}+\omega+1=0$. The dual $C_{\lambda}$ of the elliptic curve

$$
E_{\lambda}: x^{3}+y^{3}+z^{3}-3 \lambda x y z=0,
$$

is a 9-cuspidal sextic curve, (i.e. a sextic curve with 9 cusps) and conversely any 9 -cuspidal sextic curve is obtained in that way. The curve $C_{\lambda}$ is:

$$
\begin{aligned}
C_{\lambda}: & \left(x^{6}+y^{6}+z^{6}\right)+2\left(2 \lambda^{3}-1\right)\left(x^{3} y^{3}+x^{3} z^{3}+y^{3} z^{3}\right) \\
& -6 \lambda^{2} x y z\left(x^{3}+y^{3}+z^{3}\right)-3 \lambda\left(\lambda^{3}-4\right) x^{2} y^{2} z^{2}=0 .
\end{aligned}
$$

The images by the dual map of the 9 inflection points of $E_{\lambda}$ are the 9 cusps of $C_{\lambda}$. The set $\mathcal{P}_{9}$ of the 9 cusps $p_{1}, \ldots, p_{9}$ is

$$
\begin{array}{lll}
p_{1}=(\lambda: 1: 1), & p_{4}=\left(\lambda: \omega: \omega^{2}\right), & p_{7}=\left(\lambda: \omega^{2}: \omega\right) \\
p_{2}=(1: \lambda: 1), & p_{5}=\left(\omega^{2}: \lambda: \omega\right), & p_{8}=\left(\omega: \lambda: \omega^{2}\right) \\
p_{3}=(1: 1: \lambda), & p_{6}=\left(\omega: \omega^{2}: \lambda\right), & p_{9}=\left(\omega^{2}: \omega: \lambda\right) .
\end{array}
$$

When $\lambda$ varies, the closure of the set of points $p_{j}$ is a line, denoted by $L_{j}$; we obtain in that way a set $\mathcal{L}_{9}$ of 9 lines. Dually, the points on $L_{j}$ correspond to the pencil of lines meeting in the inflection point (corresponding to $p_{j}$ ) of the elliptic curve $E_{\lambda}$. One can check moreover that the line $L_{j}$ is the tangent line to the cusp $p_{j}$. Let $\mathcal{P}_{12}$ be the intersection points set of the lines in $\mathcal{L}_{9}$.

Theorem 11. The set $\mathcal{P}_{12}$ has cardinality 12 . The pair $\left(\mathcal{P}_{12}, \mathcal{L}_{9}\right)$ forms a $\left(12_{3}, 9_{4}\right)$-configuration which is the dual Hesse configuration. The set $\mathcal{C}_{12}$ of conics containing 6 points in $\mathcal{P}_{9}$ has cardinality 12 ; each conic of $\mathcal{C}_{12}$ is 
smooth. The pair of sets $\left(\mathcal{P}_{9}, \mathcal{C}_{12}\right)$ of points and conics form a $\left(9_{8}, 12_{6}\right)$ configuration.

More precisely, the union of the pairwise intersections of the 12 conics in $\mathcal{C}_{12}$ is $\mathcal{P}_{9} \cup \mathcal{P}_{12}$. The intersections between the conics are transverse. Moreover, each point of $\mathcal{P}_{12}$ is contained in exactly two conics. A conic $C$ in $\mathcal{C}_{12}$ meets 9 conics of $\mathcal{C}_{12}$ in 4 points of $\mathcal{P}_{9}$ and the two remaining conics in 3 points of $\mathcal{P}_{9}$ and one point of $\mathcal{P}_{12}$.

Proof. By a computer search, the 12 conics are:

$$
\begin{aligned}
& C_{1,2,3,4,5,6}: x^{2}+(\lambda+1)\left(\omega x y+\omega^{2} x z+y z\right)+\omega^{2} y^{2}+\omega z^{2}=0, \\
& C_{1,2,3,7,8,9}: x^{2}+(\lambda+1)\left(\omega^{2} x y+\omega x z+y z\right)+\omega y^{2}+\omega^{2} z^{2}=0, \\
& C_{1,2,4,5,7,8}: x y-\lambda z^{2}=0, \\
& C_{1,2,4,6,8,9}: x^{2}+(\omega \lambda+1)(x y+\omega x z+\omega y z)+y^{2}+\omega^{2} z^{2}=0, \\
& C_{1,2,5,6,7,9}: x^{2}+\left(\omega^{2} \lambda+1\right)\left(x y+\omega^{2} x z+\omega^{2} y z\right)+y^{2}+\omega z^{2}=0, \\
& C_{1,3,4,5,8,9}: x^{2}+\left(\omega \lambda+\omega^{2}\right)(x y+y z+\omega x z)+\omega y^{2}+z^{2}=0, \\
& C_{1,3,4,6,7,9}:-\lambda y^{2}+x z=0, \\
& C_{1,3,5,6,7,8}: x^{2}+\left(\omega^{2} \lambda+\omega\right)\left(x y+y z+\omega^{2} x z\right)+\omega^{2} y^{2}+z^{2}=0, \\
& C_{2,3,4,5,7,9}: x^{2}+\left(\lambda+\omega^{2}\right)\left(x y+x z+\omega^{2} y z\right)+\omega y^{2}+\omega z^{2}=0, \\
& C_{2,3,4,6,7,8}: x^{2}+(\lambda+\omega)(x y+x z+\omega y z)+\omega^{2}\left(y^{2}+z^{2}\right)=0, \\
& C_{2,3,5,6,8,9}: \lambda x^{2}-y z=0, \\
& C_{4,5,6,7,8,9}: x^{2}+(\lambda+1)(x y+x z+y z)+y^{2}+z^{2}=0,
\end{aligned}
$$

where the index $i, j, \ldots, n$ of the conic $C_{i, j, \ldots, n}$ means that this conic contains the 6 points $p_{s}, s \in\{i, j, \ldots, n\}$. It is easy to see that the points in $\mathcal{P}_{9}$ are in general position: no line contains 3 cusps, thus the conics are smooth. From the data of the conics and the knowledge of the points in $\mathcal{P}_{9}$ they contain, one can check the assertions about the configuration of the 12 conics and the 9 points. If one renumbers the 12 conics by their order $C_{1}, \ldots, C_{12}$ from the top to bottom of the above list, one obtains that the pair of (indexes of) conics which have an intersection point not in $\mathcal{P}_{9}$ are

$$
\begin{aligned}
& (1,2),(1,12),(2,12),(3,7),(3,11),(4,8), \\
& (4,9),(5,6),(5,10),(6,10),(7,11),(8,9),
\end{aligned}
$$

and correspondingly, the 12 points are

$$
\begin{aligned}
& (1: 1: 1),\left(\omega: \omega^{2}: 1\right),\left(\omega^{2}: \omega: 1\right),(1: 0: 0),(0: 1: 0),\left(\omega^{2}: 1: 1\right), \\
& \left(1: \omega^{2}: 1\right),(\omega: 1: 1),(1: \omega: 1),\left(\omega^{2}: \omega^{2}: 1\right),(0: 0: 1),(\omega: \omega: 1) .
\end{aligned}
$$

respectively. One can check easily that these 12 points in $\mathcal{P}_{12}$ are the intersection points of the lines in $\mathcal{L}_{9}$, which lines form the dual Hesse arrangement (see Section 2.4). By Bézout's Theorem, the intersections between the conics are transverse.

Let $q \in \mathcal{P}_{9}$ and define $\mathcal{P}_{q}=\mathcal{P}_{9} \backslash\{q\}$.

Theorem 12. The set $\mathcal{C}_{q}$ of conics containing the point $q$ has cardinality 8. The set of points $\mathcal{P}_{q}$ and the set of conics $\mathcal{C}_{q}$ form an $8_{5}$-configuration: each point is on 5 conics and each conic contains 5 of the points in $\mathcal{P}_{q}$. 
For each conic $C$ in $\mathcal{C}_{q}$ there exists a unique conic $C^{\prime} \in \mathcal{C}_{q}$ such that there is a unique point in the intersection of $C$ and $C^{\prime}$ which is not in $\mathcal{P}_{q}$.

Proof. That can be checked directly from the datas in the proof of Proposition 11 .

Let $X_{\lambda}$ be the minimal desingularization of the double cover branched over the sextic curve $C_{\lambda}$ with 9 cusps. We denote by $\eta: X_{\lambda} \rightarrow \mathbb{P}^{2}$ the natural map and we denote by $A_{j}, A_{j}^{\prime}$ the two $(-2)$-curves in $X$ above the point $p_{j}$ in $\mathcal{P}_{9}$. The curves $A_{j}, A_{j}^{\prime}, j \in\{1, \ldots, 9\}$ form a $9 \mathbf{A}_{2}$-configuration, which we call the natural $9 \mathbf{A}_{2}$-configuration. We have

Lemma 13. The strict transform by the map $\eta: X_{\lambda} \rightarrow \mathbb{P}^{2}$ of a conic $C \in \mathcal{C}_{12}$ is the union of two disjoint $(-2)$-curves $\theta_{C}, \theta_{C}^{\prime}$.

Let $C, D$ be two conics in $\mathcal{C}_{12}$. Suppose that $C$ and $D$ meet in 4 points in $\mathcal{P}_{9}$. Then the $(-2)$-curves $\theta_{C}, \theta_{C}^{\prime}, \theta_{D}, \theta_{D}^{\prime}$ are disjoint.

Suppose that $C$ and $D$ meet in 3 points in $\mathcal{P}_{9}$. Then, up to exchanging $\theta_{D}$ and $\theta_{D}^{\prime}$, the curves $\theta_{C}, \theta_{D}, \theta_{C}^{\prime}, \theta_{D}^{\prime}$ form a $2 \mathbf{A}_{2}$-configuration.

Proof. Rather than performing a double cover and taking the resolution of surface singularities, we perform one blow-ups at each cusp $q \in \mathcal{P}_{9}$ of $C_{\lambda}$, so that the branch locus is smooth and tangent to the exceptional curve $E$ (see also Figure 3.1). On the double cover, the reduced image inverse of the curve $E$ is the union of two (-2)-curves on the smooth K3 surface $X_{\lambda}$.

By that local computation, we see that for $C \in \mathcal{C}_{12}$, the curves $\theta_{C}, \theta_{C^{\prime}}$ are disjoint (the strict transform $\bar{C}$ of $C$ under the blow-up map do not meet the branch locus).

Suppose $C$ and $D$ meet in 4 points in $\mathcal{P}_{9}$. The intersection being transverse, each strict transforms $\bar{C}, \bar{D}$ of the curves $C, D$ under the 3 blow-ups at each cusps is the union of two disjoint curves not meeting the branch curve and the 4 curves in $\bar{C}, \bar{D}$ are disjoint.

If $C$ and $D$ meet in 3 points in $\mathcal{P}_{9}$ then they meet transversely at a unique point not in $\mathcal{P}_{9}$. Then taking the above notations, we have this time $\bar{C} \bar{D}=1$, so that the last assertion holds.

Let $\mathcal{P}_{q}$ and $\mathcal{C}_{q}$ as above. Using Theorem [12 and Lemma 13, we get:

Corollary 14. The strict transform by $\eta$ of the 8 conics in $\mathcal{C}_{q}$ forms an $8 \mathbf{A}_{2}$-configuration.

For each point $q=p_{j}, j \in\{1, \ldots, 9\}$, we denote by $\mathcal{A}_{j}^{\prime}$ the corresponding $8 \mathbf{A}_{2}$-configuration on $X_{\lambda}$. In order to obtain new $9 \mathbf{A}_{2}$-configurations, one needs to find other $\mathbf{A}_{2}$-configurations. This will be done in the next section by using singular quartics instead of conics.

Remark 15. Using a computer, we found eight $8 \mathbf{A}_{2}$-configurations in the set of $32(-2)$-curves which is the union of the two $8 \mathbf{A}_{2}$-configurations $\mathcal{A}_{j}^{\prime}$ and $\left\{A_{1}, A_{1}^{\prime}, \ldots, A_{9}, A_{9}^{\prime}\right\} \backslash\left\{A_{j}, A_{j}^{\prime}\right\}$. However one can compute that the orthogonal complement of 6 of them are lattices with no $(-2)$-classes, thus 
one cannot complete these 6 configurations into $9 \mathbf{A}_{2}$-configurations. The $32(-2)$-curves can be realized as lines in a projective model of $X_{\lambda}$, see Proposition 19.

3.2. Nine new $9 \mathbf{A}_{2}$-configurations. Let $p_{j} \in \mathcal{P}_{9}$ be one of the 9 cusp singularities of the sextic $C_{\lambda}$.

Theorem 16. There exists a unique quartic curve $Q_{j}$ passing through the 9 points in $\mathcal{P}_{9}$, with a unique singularity at the point $p_{j}$. The singularity has multiplicity 3 , it is of type $\mathbf{D}_{5}$ : it has two tangents, one branch is smooth while the other branch is a cuspidal singularity. The tangent at the cusp of $Q_{j}$ is also the tangent to the cuspidal singularity of the sextic $C_{\lambda}$ at $p_{j}$.

The curve $Q_{j}$ has geometric genus 0 . Its strict transform denoted by $\eta$ on $X_{\lambda}$ is the union of two (-2)-curves $\gamma_{j}, \gamma_{j}^{\prime}$ which form an $\mathbf{A}_{2}$-configuration. The curves $\gamma_{j}, \gamma_{j}^{\prime}$ and the 16 curves in $\mathcal{A}_{j}^{\prime}$ form a $9 \mathbf{A}_{2}$-configuration $\mathcal{A}_{j}$.

Proof. We give in the Appendix the equations of the 9 curves $Q_{j}, j \in$ $\{1, \ldots, 9\}$. These curves have been constructed using the LinSys program by C. Rito which enables to find curves of given degree with prescribed singularities and given tangencies at a set of points in the plane. Conversely, one can check that the singularity of $Q_{j}$ at $p_{j}$ has multiplicity 3 , is resolved by one blow-up, with the exceptional divisor meeting the strict transform in two points, one of multiplicity 2 .

The curve $Q_{j}$ has genus 0, (see e.g. [12, Chapter 4, Section 2]). By Bézout's Theorem, the intersections of the quartic $Q_{j}$ with the 8 conics in $\mathcal{C}_{12}$ that contain $p_{j}$ are transverse, so that the curves in $\mathcal{A}_{j}^{\prime}$ are disjoint from $\gamma_{j}, \gamma_{j}^{\prime}$ and we thus get a $9 \mathbf{A}_{2}$-configuration. See Figure 3.1 for the behavior of the quartic curve $Q_{j}$ under the double cover.

Figure 3.1. Behavior of the quartic $Q_{j}$ under the double cover

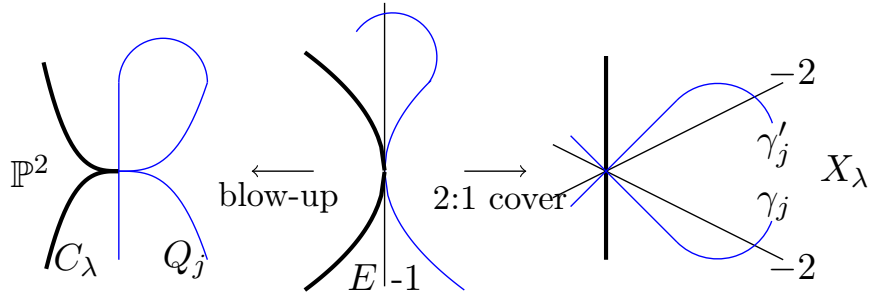

Remark 17. In Section 4.3, we study some automorphisms of $X_{\lambda}$. We obtain that the nine above $9 \mathbf{A}_{2}$-configurations are in the same orbit under the action by 3-torsion of the Mordell-Weil group of a fibration of $X_{\lambda}$.

4. Projective, Hessian and Weierstrass models of $X_{\lambda}$

4.1. The natural elliptic fibration of $X_{\lambda}$. Let $D_{2}$ be the big and nef divisor on $X_{\lambda}$ which is the pull back of a line in $\mathbb{P}^{2}$. For $\lambda$ generic, the 
divisors $D_{2}, A_{1}, A_{1}^{\prime} \ldots, A_{9}, A_{9}^{\prime}$ form a $\mathbb{Q}$-basis $\mathcal{B}_{\mathbb{Q}}$ of $\mathrm{NS}\left(X_{\lambda}\right)_{/ \mathbb{Q}}$; they generate an index $3^{6}$ sub-lattice of $\mathrm{NS}\left(X_{\lambda}\right)$ (see also Lemma 8).

Let $\mu: Y_{\lambda} \rightarrow \mathbb{P}^{2}$ be the blow-up of the plane at the 9 cusps of the sextic curve $C_{\lambda}$ and let $E_{1}, \ldots, E_{9}$ be the exceptional curves over $p_{1}, \ldots, p_{9}$. The strict transform by $\mu$ of the curve $C_{\lambda}$ is the smooth genus 1 curve

$$
\bar{C}_{\lambda}=\mu^{*} C_{\lambda}-2 \sum_{j=1}^{9} E_{i}, \text { such that } \bar{C}_{\lambda}^{2}=0 .
$$

The surface $X_{\lambda}$ is the double cover of $Y_{\lambda}$ branched over $\overline{C_{\lambda}}$; we denote by

$$
\eta^{\prime}: X_{\lambda} \rightarrow Y_{\lambda}
$$

the double cover morphism (so that $\eta^{\prime *} E_{j}=A_{j}+A_{j}^{\prime}$ ) and by $F_{o}$ the ramification locus, so that $2 F_{o}=\eta^{*} \bar{C}_{\lambda}$. Since $2 F_{o}=\eta^{*} \bar{C}_{\lambda} \equiv 6 D_{2}-2 \sum_{j=1}^{9}\left(A_{j}+A_{j}^{\prime}\right)$, we get

$$
F_{o} \equiv 3 D_{2}-\left(\sum_{j=1}^{9} A_{j}+A_{j}^{\prime}\right) .
$$

Let $L \hookrightarrow \mathbb{P}^{2}$ be a line; the curve $C_{\lambda}$ belongs to the linear system

$$
\delta=\left|6 L-2 \sum_{j=1}^{9} p_{j}\right|
$$

of sextic curves with a double point at points in $\mathcal{P}_{9}$. One computes that this linear system is 1 dimensional. Moreover there exists a unique cubic curve $\mathrm{Ca}(\lambda)$ (called the Cayleyan curve, see [1]) that contains the 9 points in $\mathcal{P}_{9}$, which is

$$
\mathrm{Ca}(\lambda): x^{3}+y^{3}+z^{3}-\frac{\left(\lambda^{3}+2\right)}{\lambda} x y z=0
$$

so that $2 \mathrm{Ca}(\lambda) \in \delta$. The linear system $\delta$ lifts to a base point free linear system $\delta^{\prime}$ on $Y_{\lambda}$ with $\bar{C}_{\lambda} \in \delta^{\prime}$. The linear system $\delta^{\prime}$ defines a morphism $\varphi^{\prime}: Y_{\lambda} \rightarrow \mathbb{P}^{1}$ and induces an elliptic fibration

$$
\varphi: X_{\lambda} \rightarrow \mathbb{P}^{1}
$$

for which $F_{o}$ is a fiber, and which we call the natural fibration.

Let $p, q$ be the images by $\varphi$ of the strict transforms in $X_{\lambda}$ of $\mathrm{Ca}(\lambda)$ and $C_{\lambda}$. In fact the surface $X_{\lambda}$ is the fiber product of the fibration $\varphi^{\prime}$ and the quadratic transformation $\mathbb{P}^{1} \rightarrow \mathbb{P}^{1}$ branched at $p, q$. Indeed both maps $X_{\lambda} \rightarrow Y_{\lambda}$ and $Y_{\lambda} \times_{\mathbb{P}^{1}} \mathbb{P}^{1}$ has the same branch locus in the rational surface $Y_{\lambda}$.

The curves $A_{1}, A_{1}^{\prime}, \ldots, A_{9}, A_{9}^{\prime}$ are sections of $\varphi$, and one can check that the curves $\gamma_{1}, \gamma_{1}^{\prime}, \ldots, \gamma_{9}, \gamma_{9}^{\prime}$ are also sections (see Figure 3.1).

Theorem 18. The fibration $\varphi$ contracts the $24(-2)$-curves $\Theta_{j}, j \in\{1, \ldots, 24\}$ which are above the 12 conics $\mathcal{C}_{12}$. The singular fibers of $\varphi$ are 8 fibers of type $\tilde{\mathbf{A}}_{2}$, each singular fiber is the union of 3 curves $\Theta_{j}$. For $\lambda$ generic, the fibration $\varphi$ has fibers with non-constant moduli and the Mordell-Weil group of the fibration $\varphi$ is $\mathbb{Z} \times(\mathbb{Z} / 3 \mathbb{Z})^{2}$.

Proof. The following four sextic curves

$$
\begin{aligned}
& C_{123456}+C_{123789}+C_{456789}, C_{124578}+C_{134679}+C_{235689}, \\
& C_{124689}+C_{135678}+C_{234579}, C_{125679}+C_{134589}+C_{234678}
\end{aligned}
$$


belong to the linear system $\delta$ of sextic curves that have multiplicity 2 at the points in $\mathcal{P}_{9}$; actually their singularities are nodes. By the results in the proof of Theorem 11, the strict transform to $Y_{\lambda}$ of the above 4 sextic form 4 fibers of type $\tilde{\mathbf{A}}_{2}$, which lies in the étale locus of $\eta$. Their strict transform on $X_{\lambda}$ is therefore the union of eight fibers of type $\tilde{\mathbf{A}}_{2}$. A fiber of type $\tilde{\mathbf{A}}_{2}$ contributes to 3 in the Euler characteristic of $X_{\lambda}$, which is equal to 24 . Since there are $8 \tilde{\mathbf{A}}_{2}$ singular fibers, the fibration has no other singular fibers. The 24 curves $\Theta_{j}$ above the 12 conics are in the fibers, thus are contracted by $\varphi$.

The strict transform $\mathrm{He}(\lambda)$ on $X_{\lambda}$ of $\mathrm{Ca}(\lambda)$ is smooth, of genus 1 (we will see that this is the Hessian of the curve $E_{\lambda}$, thus the notation; see also Remark 25). Since $\operatorname{He}(\lambda) \cdot F_{o}=0$, we have that $\operatorname{He}(\lambda) \equiv F_{o}$. The curve $F_{o}$ is isomorphic to $E_{\lambda}$. For generic $\lambda$, the curves $\operatorname{Ca}(\lambda)$ and $E_{\lambda}$ have distinct $j$-invariants, thus the fibers of $\varphi$ have a non-constant moduli. Since the fibration is not isotrivial, results of Shioda (see [29, Corollary 1.5]) apply and tell that the Mordell-Weil group of sections of $\varphi: X_{\lambda} \rightarrow \mathbb{P}^{1}$ has rank $1=19-(2+8(3-1))$.

In fact, elliptic fibrations of K3 surfaces are classified by Shimada in [27]. A table with the 3278 possible cases is available in [28]. Our fibration is case number 2373 in that table, where one can find moreover that the torsion part of its Mordell-Weil group is isomorphic to $(\mathbb{Z} / 3 \mathbb{Z})^{2}$.

4.2. Two polarizations and a degree 8 projective model. The divisor

$$
D_{14}=4 D_{2}-\left(\sum_{j=1}^{9} A_{j}+A_{j}^{\prime}\right)
$$

is linearly equivalent to $D_{2}+F$ ( $F$ a fiber of $\varphi$ ) and is effective. Let us define $D_{8}=D_{14}-\left(A_{1}+A_{1}^{\prime}\right)$.

Proposition 19. The divisors $D_{8}$ and $D_{14}$ are ample of square $D_{8}^{2}=8$, $D_{14}^{2}=14$. The linear system $\left|D_{8}\right|$ is base point free, non-hyperelliptic, and defines an embedding $X_{\lambda} \hookrightarrow \mathbb{P}^{5}$ as a degree 8 surface. For $d \in \mathbb{N}^{*}$, let $n_{d}$ be the number of $(-2)$-curves of degree $d$ for $D_{8}$. The series $\sum n_{d} T^{d}$ begins with

$$
32 T+20 T^{2}+334 T^{4}+576 T^{5}+880 T^{6}+8640 T^{7}+17784 T^{8} \ldots,
$$

in particular $X_{\lambda}$ contains 32 lines and 20 conics.

Proof. Let $B$ be a $(-2)$-curve such that $D_{14} B \leq 0$. Since $D_{2}$ is effective and $D_{2}^{2}>0$, one has $D_{2} B \geq 0$, moreover since $F$ is a fiber, $F B \geq 0$ and we must have $D_{2} B=0=F B$. That implies that $B$ is an irreducible component of a singular fiber, ie $B=\Theta_{j}$ for some $j \in\{1,, \dot{2} \dot{4}\}$. But since $D_{2} \Theta_{j}=2$ for all $j$, such a curve $B$ cannot exist, thus $D_{14}$ is ample.

Let us prove that $D_{8}$ is ample. We have

$$
\gamma_{1}+\gamma_{1}^{\prime} \equiv 4 D_{2}-2\left(A_{1}+A_{1}^{\prime}\right)-\sum_{j=1}^{9}\left(A_{j}+A_{j}^{\prime}\right),
$$

thus $D_{8} \equiv A_{1}+A_{1}^{\prime}+\gamma_{1}+\gamma_{1}^{\prime}$ and the divisor $D_{8}$ is effective. We check that $D_{8} A_{1}=D_{8} A_{1}^{\prime}=D_{8} \gamma_{1}=D_{8} \gamma_{1}^{\prime}=1$ and $D_{8}^{2}=8$, therefore $D_{8}$ is nef and big. 
Suppose that there is a $(-2)$-curve $B$ on $X_{\lambda}$ such that $D_{8} B=0$. Then by the above expression of $D_{8}$, one has $A_{1} B=A_{1}^{\prime} B=0$. Let $L \hookrightarrow \mathbb{P}^{2}$ be a line. For $j \in\{2, \ldots, 9\}$, let us consider the linear system

$$
\delta_{j}=\left|4 L-\left(p_{1}+p_{j}+\sum_{j=1}^{9} p_{k}\right)\right|
$$

of the quartic curves that go through the points in $\mathcal{P}_{9}$ and with multiplicity 2 at $p_{1}$ and $p_{j}$. Using LinSys, one can compute that for each $j>1$, the linear system $\delta_{j}$ is a pencil of curves and the base points set is $\mathcal{P}_{9}$. Moreover, the generic element $\vartheta_{j}$ of $\delta_{j}$ is an irreducible curve of geometric genus 1 which cuts $C_{\lambda}$ in $\mathcal{P}_{9}$ and two more points. Thus we obtain that for each $j>1$, the strict transform of $\vartheta_{j}$ is an irreducible curve $\Gamma_{j}$ such that

$$
D_{8} \equiv \Gamma_{j}+A_{j}+A_{j}^{\prime} \text { and } \Gamma_{j}^{2}=2 .
$$

Since $D_{8} B=0$, we obtain $A_{j} B=A_{j}^{\prime} B=0$ for all $j \in\{1, \ldots, 9\}$. Since the orthogonal of the classes $A_{j}, A_{j}^{\prime}, j \in\{1, \ldots, 9\}$ (on which $B$ belongs) is generated by $D_{2}$, the class of $B$ must be a multiple of $D_{2}$ and have positive square, which is absurd. Therefore $D_{8}$ is ample.

Suppose that there is a fiber $F^{\prime}$ such that $D_{8} F^{\prime} \in\{1,2\}$. Observe that by using the expression for $D_{8}$, we get that $F^{\prime} \Gamma_{j}=0,1,2$. If $F^{\prime} \Gamma_{j}=0$, then $\Gamma_{j}$ is contained in a fiber of the fibration determined by $F^{\prime}$, but this is not possible since $\Gamma_{j}^{2}=2$. If $F^{\prime} \Gamma_{j}=1$, then $\Gamma_{j}$ is a section of the fibration so is a rational curve, but again this is not possible. If $F^{\prime} \Gamma_{j}=2$ (we can assume that this holds for all $j$, otherwise we are in a previous case), then $F^{\prime}$ is in the orthogonal complement of the $A_{j}, A_{j}^{\prime}$ but this is not possible since this is generated by $L$, which is of square 2 . Therefore there are no such fiber $F^{\prime}$ and using [24], we obtain that the linear system $\left|D_{8}\right|$ is base-point free and gives an embedding of $X_{\lambda}$.

With respect to the divisor $D_{8}$, the degrees of the curves $A_{1}, A_{1}^{\prime}, \gamma_{1}, \gamma_{1}^{\prime}$ equal 2 and the degrees of curves $A_{i}, A_{i}^{\prime}, i \geq 2$ is 1 . For the assertions on the number of rational curves of degree $d \leq 8$ we used an algorithm (see e.g. [20]), which computes the classes of $(-2)$-curves in $\operatorname{NS}\left(X_{\lambda}\right)$ of given degrees with respect to a fixed ample class.

Proceeding in a similar way as in the proof of Proposition [19, we obtain:

Proposition 20. Let $i, j \in\{1, \ldots, 9\}, i \neq j$. The divisor

$$
D_{i, j}=D_{14}-\left(A_{i}+A_{i}^{\prime}+A_{j}+A_{j}^{\prime}\right)
$$

is nef of square 2 and the linear system $\left|D_{i, j}\right|$ is base point free.

One can compute that the intersection with $D_{i j}$ is 0 for the 10 curves $\theta_{i j k l m n}, \theta_{i j k l m n}^{\prime}$ (where $\{k, l, m, n\} \subset\{1, \ldots, 9\}$ is a set of 4 elements such that the conic $C_{i j k l m n}$ exists), and for the $(-2)$-curve which is the strict transform on $X_{\lambda}$ of the line through cusps $p_{i}, p_{j}$.

\subsection{A Hessian model of the natural fibration of $X_{\lambda}$.}


4.3.1. The generic fiber of the elliptic fibration $\varphi$ and 18 rational points. Let $f_{\lambda}$ be the equation of the 9 cuspidal sextic $C_{\lambda}$ which is the dual of $E_{\lambda}$, and let $c_{\lambda}$ be the equation of the Cayleyan elliptic curve $\mathrm{Ca}(\lambda)$ (see equation (4.1)), the unique cubic that goes through the 9 cusps of the sextic curve $C_{\lambda}$.

We recall (see Section 4.1) that $Y_{\lambda}$ is the blow-up of the plane at the 9 points in $\mathcal{P}_{9}$; it has a natural elliptic fibration $\varphi^{\prime}$, coming from the pencil of sextic curves which have double points at the 9 points in $\mathcal{P}_{9}$, pencil which is generated by $C_{\lambda}$ and $2 \mathrm{Ca}(\lambda)$. A singular model of $Y_{\lambda}$ is therefore obtained as the surface in $\mathbb{P}^{1} \times \mathbb{P}^{2}$ with equation $u f_{\lambda}-v c_{\lambda}^{2}=0$, where $u, v$ are the coordinates of $\mathbb{P}^{1}$. The projection onto $\mathbb{P}^{1}$ induces the fibration $\varphi^{\prime}: Y_{\lambda} \rightarrow \mathbb{P}^{1}$. A singular model of the $K 3$ surface $X_{\lambda}$ is the surface $X_{\lambda}^{\text {sing }}$ in $\mathbb{P}^{1} \times \mathbb{P}^{2}$ with equation $u^{2} f_{\lambda}-v^{2} c_{\lambda}^{2}=0$; again the projection onto $\mathbb{P}^{1}$ induces the natural fibration $X_{\lambda}^{\text {sing }} \rightarrow \mathbb{P}^{1}$, where the generic fibers are 9-nodal sextic curves.

In order to obtain a smooth model of $X_{\lambda}$, let us consider the linear system $L_{4}\left(\mathcal{P}_{9}\right)$ of quartics that contain the 9 cusps. The linear system $L_{4}\left(\mathcal{P}_{9}\right)$ has (projective) dimension 5 and defines a rational map $\phi: \mathbb{P}^{2} \rightarrow \mathbb{P}^{5}$ not defined on $\mathcal{P}_{9}$. One computes that the image of $X_{\lambda}^{\text {sing }}$ by the rational map

$$
\left(i_{d}, \phi\right): \mathbb{P}^{1} \times \mathbb{P}^{2} \rightarrow \mathbb{P}^{1} \times \mathbb{P}^{5}
$$

is a smooth model of $X_{\lambda}$; from Section 4.1, the images of the cusps are the $18(-2)$-curves on $X_{\lambda}$ forming a $9 \mathbf{A}_{2}$-configuration. Taking the generic point over $\mathbb{P}^{1}$, one get a smooth genus 1 curve in $\mathbb{P}_{/ \mathbb{Q}(t)}^{5}$ (where $\left.t=\frac{u}{v}\right)$. That curve $E_{K 3}$ has naturally 18 rational points, corresponding to the $18(-2)$-curves. Using Magma, we computed a Hessian model $E_{K 3} \hookrightarrow \mathbb{P}_{/ \mathbb{Q}(t)}^{2}$, which is

Theorem 21. A model of the generic fiber of the fibration $X_{\lambda} \rightarrow \mathbb{P}^{1}$ is

$$
E_{K 3} \quad x^{3}+y^{3}+z^{3}+\frac{\lambda^{3}\left(t^{2}+3\right)-4 t^{2}}{\lambda^{2}\left(t^{2}-1\right)} x y z=0 .
$$

The elliptic curve $E_{K 3}$ contains the 9 obvious 3 -torsion points

$$
\begin{aligned}
& Q_{1}=(0:-1: 1), Q_{2}=(-1: 0: 1), Q_{3}=(-1: 1: 0), \\
& Q_{4}=(0:-\omega: 1), Q_{5}=(\omega+1: 0: 1), Q_{6}=(-\omega: 1: 0), \\
& Q_{7}=(0: \omega+1: 1), Q_{8}=(-\omega: 0: 1), Q_{9}=(\omega+1: 1: 0)
\end{aligned}
$$

(where $\omega^{2}+\omega+1=0$; we take $Q_{1}$ as the neutral element) and the following 9 points

$$
\begin{aligned}
& P_{1}=(-2 t: \lambda(t+1): \lambda t+\lambda), \\
& P_{2}=(\lambda(t-1):-2 t: \lambda t+\lambda), \\
& P_{3}=(-\lambda t-\lambda:-\lambda t+\lambda: 2 t), \\
& P_{4}=((2 \omega+2) t: \lambda(\omega t+\omega): \lambda t+\lambda) \\
& P_{5}=(\lambda(\omega+1)(-t+1):-2 \omega t: \lambda t+\lambda), \\
& P_{6}=((\omega+1) \lambda(t+1): \omega \lambda(-t+1): 2 t), \\
& P_{7}=(-2 \omega t:-\lambda(\omega+1)(t+1): \lambda t-\lambda), \\
& P_{8}=(\lambda \omega(t-1):(2 \omega+2) t: \lambda t+\lambda), \\
& P_{9}=(-\lambda \omega(t-1):(\omega+1) \lambda(t-1): 2 t) .
\end{aligned}
$$


Together, these 18 points are the above-mentioned rational points of $E_{K 3 / \mathbb{Q}(\omega, t)}$ corresponding to the 18 sections of the fibration of $X_{\lambda}$.

Remark 22. For the neutral element of $E_{K 3}$, let us choose $Q_{1}$. One can check that the point $P_{k}$ is the translate of $P_{1}$ by the 9 torsion point $Q_{k}$ $(k \in\{1, \ldots, 9\})$, i.e. $P_{k}=P_{1}+Q_{k}$.

4.3.2. A smooth model of $X_{\lambda}$ in $\mathbb{P}^{1} \times \mathbb{P}^{2}$. By taking the homogenization of the generic fiber $E_{K 3}$ in (4.2), we get a natural model of the K3 surface $X_{\lambda}$ as

$$
\lambda^{2}\left(u^{2}-v^{2}\right)\left(x^{3}+y^{3}+z^{3}\right)+\left(\lambda^{3}\left(u^{2}+3 v^{2}\right)-4 u^{2}\right) x y z=0 .
$$

in the space $\mathbb{P}^{1} \times \mathbb{P}^{2}$ (with coordinates $u, v ; x, y, z$, where $t=\frac{u}{v}$ ). That model is smooth, and the generic fibers are smooth cubic curves, by contrast with the previous model $X_{\lambda}^{\text {sing }}$. We denote by $(P)$ the section in $X_{\lambda}$ corresponding to the points $P \in E_{K 3}$. Using Magma, it is then possible to obtain the equations of the $(-2)$-curves (also sections) $A_{j}=\left(Q_{j}\right)$, resp. $A_{j}^{\prime}=\left(P_{i}\right)$, which are on $X_{\lambda} \hookrightarrow \mathbb{P}^{1} \times \mathbb{P}^{2}$. We can check that:

Lemma 23. The 9 curves $A_{j}+A_{j}^{\prime}(j \in\{1, \ldots, 9\})$ form a $9 \mathbf{A}_{2}$-configuration.

Proof. We use the equations of the (-2)-curves $A_{j}, A_{j}^{\prime}$ in the model $X_{\lambda} \subset$ $\mathbb{P}^{1} \times \mathbb{P}^{2}$ to check that $A_{j} A_{j}^{\prime}=1$ and $A_{j} A_{k}^{\prime}=A_{j} A_{k}=A_{j}^{\prime} A_{k}^{\prime}=0$ for $k \neq j$. In fact, one already knows that 3 -torsion sections are disjoint by [15, VII, Proposition 3.2] (thus the sections $A_{j}^{\prime}$, being translated of the group of 3torsion sections, are also disjoint).

One can check moreover that the 9 intersection points of $A_{j}$ with $A_{j}^{\prime}$ for $i=1, \ldots, 9$ are on the fiber over 0 of the fibration $\varphi$, fiber which is isomorphic to $E_{\lambda}$. Using the addition law on the elliptic curve $E_{K 3}$, one can find other points of $E_{K 3}$, and therefore sections of $\varphi$. By example the following points

$$
\begin{aligned}
& R_{1}=(-2 t: \lambda(t-1): \lambda t+\lambda), \\
& R_{2}=(\lambda(t+1):-2 t: \lambda t-\lambda), \\
& R_{3}=(-\lambda t+\lambda:-\lambda t-\lambda: 2 t), \\
& R_{4}=((2 \omega+2) t: \lambda \omega(t-1): \lambda t+\lambda), \\
& R_{5}=(-\lambda(\omega+1)(t+1):-2 \omega t: \lambda t-\lambda), \\
& R_{6}=((\omega+1) \lambda(t-1):-\omega \lambda(t+1): 2 t), \\
& R_{7}=(-2 \omega t: \lambda(\omega+1)(-t+1): \lambda t+\lambda), \\
& R_{8}=(\lambda \omega(t+1):(2 \omega+2) t: \lambda t-\lambda), \\
& R_{9}=(-\omega \lambda t+\omega \lambda:(\omega+1)(\lambda t+\lambda): 2 t),
\end{aligned}
$$

are the points $R_{i}=-P_{1}+Q_{i}, i \in\{1, \ldots, 9\}$. We have

Lemma 24. The 9 curves $A_{i},\left(R_{i}\right), i=1, \ldots, 9$, form a $9 \mathbf{A}_{2}$-configurations.

Proof. The curves $A_{i}$ (resp. $\left.\left(R_{i}\right)\right)$ are images of the curves $A_{i}^{\prime}$ (resp. $A_{i}$ ) by the translation by $-A_{1}^{\prime}$ and we already know that the curves $A_{i}, A_{i}^{\prime}$ form a $9 \mathbf{A}_{2}$-configuration. 
We already know that the fiber at 0 of the elliptic K3 surface $\varphi: X_{\lambda} \rightarrow \mathbb{P}^{1}$ is isomorphic to $E_{\lambda}$, moreover:

Remark 25. From equation 4.3, the fiber at $\infty$ of $X_{\lambda} \rightarrow \mathbb{P}^{1}$ is the elliptic curve

$$
\operatorname{He}(\lambda): x^{3}+y^{3}+z^{3}+\frac{\left(\lambda^{3}-4\right)}{\lambda^{2}} x y z=0,
$$

which is in fact the Hessian of the curve $E_{\lambda}$. The $j$-invariants of $\mathrm{Ca}(\lambda)$ and $\operatorname{He}(\lambda)$ are distinct, in particular these curves are not isomorphic. The Cayleyan curve $\mathrm{Ca}(\lambda)$ is the quotient of Hessian $\mathrm{He}(\lambda)$ of $E_{\lambda}$ by a 2-torsion point; in particular the two curves are 2-isogeneous, see [1].

4.3.3. A degree 8 non-complete intersection model in $\mathbb{P}^{5}$. One can check that the map from $X_{\lambda} \subset \mathbb{P}^{1} \times \mathbb{P}^{2}$ to $\mathbb{P}^{5}$ obtained as the product of the identity map of $\mathbb{P}^{1}$ with the Segre embedding composed with the projection to $\mathbb{P}^{5}$, is an embedding with image a degree $8 \mathrm{~K} 3$ surface in $\mathbb{P}^{5}$ defined by the following 5 equations:

$$
\begin{array}{rr}
-U_{2} U_{4}+U_{1} U_{5}, \quad-U_{2} U_{3}+U_{0} U_{5}, \quad-U_{1} U_{3}+U_{0} U_{4}, \\
\lambda^{2}\left(U_{0}^{2} U_{3}-U_{3}^{3}+U_{1}^{2} U_{4}-U_{4}^{3}\right)+\left(\lambda^{3}-4\right) U_{0} U_{1} U_{5} \\
+\lambda^{2}\left(U_{2}^{2} U_{5}+3 \lambda U_{3} U_{4} U_{5}-U_{5}^{3}\right), \\
\lambda^{2}\left(U_{0}^{3}+U_{1}^{3}+U_{2}^{3}-U_{0} U_{3}^{2}\right)+\left(\lambda^{3}-4\right) U_{0} U_{1} U_{2} \\
+\lambda^{2}\left(-U_{1} U_{4}^{2}+3 \lambda U_{0} U_{4} U_{5}-U_{2} U_{5}^{2}\right),
\end{array}
$$

in particular this is not a complete intersection (in fact, by using [2, Chapter VIII, Exercice 11], a K3 surface has a degree 8 smooth model which is not a complete intersection if and only if it has a smooth model in $\mathbb{P}^{1} \times \mathbb{P}^{2}$ of bi-degree $(2,3))$.

Using the images of the known $(-2)$-curves on $X_{\lambda}$, one finds that this surface in $\mathbb{P}^{5}$ contains at least 33 lines.

The involution $\sigma^{\prime}$ defined by $u \rightarrow\left(-u_{0}:-u_{1}:-u_{2}: u_{3}: u_{4}: u_{5}\right)$ acts on $X_{\lambda} \hookrightarrow \mathbb{P}^{5}$. Also one can check that the order 3 automorphisms

$$
\begin{aligned}
& \alpha_{1}: u \rightarrow\left(u_{1}: u_{2}: u_{0}: u_{4}: u_{5}: u_{3}\right) \\
& \alpha_{2}: u \rightarrow\left(u_{0}: \omega u_{1}: \omega^{2} u_{2}: u_{3}: \omega u_{4}: \omega^{2} u_{5}\right)
\end{aligned}
$$

act on $X_{\lambda}$ and so does the involution

$$
\beta: u \rightarrow\left(u_{0}: u_{2}: u_{1}: u_{3}: u_{5}: u_{4}\right) .
$$

The fixed point set of $\alpha_{1}$ is the union of 6 points, the fixed point set of $\beta$ is a genus 2 curve. The involution $\sigma^{\prime} \beta$ is symplectic. Using the above equations of $X_{\lambda}$ and the equations of curves $A_{1}, \ldots, A_{9}^{\prime}$ in $\mathbb{P}^{5}$, one can check that the automorphism group $G_{\mathcal{C}}$ that preserve globally the $9 \mathbf{A}_{2}$-configuration $A_{1}, \ldots, A_{9}^{\prime}$ contains the group $G_{18}$ isomorphic to $\mathbb{Z}_{3} \rtimes S_{3}$ generated by $\alpha_{1}, \alpha_{2}, \sigma^{\prime} \beta$. We prove in Section 5.3 that $G_{18}$ has index 2 in the group $G_{\mathcal{C}}$ preserving the configuration $A_{1}, \ldots, A_{9}^{\prime}$. 
4.4. A Weierstrass equation. We recall that $\omega$ is such that $\omega^{2}+\omega+1=0$. Let us prove the following result

Theorem 26. A minimal Weierstrass model of the elliptic K3 surface $X_{\lambda}$ is the elliptic curve

$$
E_{1 / \mathbb{Q}(\omega, t)}: y^{2}=x^{3}-\frac{1}{48} A x+\frac{1}{864} B,
$$

where the three polynomials $A, B, D$ in $\mathbb{Q}(\omega)(t)$ (of respective degree 8, 12 and 8) are defined in the Appendix. The 8 singular fibers $\tilde{\mathbf{A}}_{2}$ of $X_{\lambda}$ are over the 8 zeros of $D$.

Proof. A direct computations gives that the $j$-invariant of the elliptic curve $E_{K 3}$ is

$$
j=-\frac{A^{3}}{\left(\lambda^{2}\left(\lambda^{3}-1\right) D\right)^{3}},
$$

where the formulas for the polynomials $A$ and $D$ in $t$ are given in the Appendix. For any $j \notin\{0,1728\}$, the elliptic curve

$$
E_{0}(j): \quad y^{2}=x^{3}-\frac{1}{48} \frac{j}{j-1728} x+\frac{1}{864} \frac{j}{j-1728}
$$

has $j$-invariant equal to $j$. In our case, we compute that we have

$$
\frac{j}{j-1728}=\frac{A^{3}}{B^{2}}
$$

where the polynomial $B$ is also defined in the Appendix. By taking the change of variables

$$
x^{\prime}=u^{2} x, y^{\prime}=u^{3} y
$$

with $u=(B / A)^{1 / 2}$ in the equation of $E_{0}(j)$, we obtain the elliptic curve $E_{1}$. The curve $E_{1}$ has also its $j$-invariant equals to $j$, is also in Weierstrass form, but its coefficients are coprime degree 8 and 12 polynomials in $t$. The discriminant of the equation of $E_{1}$ is

$$
\Delta=-\left(\lambda^{2}\left(\lambda^{3}-1\right) D\right)^{3},
$$

where $D$ is a product of 8 degree 1 polynomials in $t$. According to [15, Table IV.3.1], the associated elliptic surface is a K3 surface with 8 singular fibers of type $\tilde{\mathbf{A}}_{2}$.

Using Magma, we finally obtain an isomorphism defined over $\mathbb{Q}(\omega, t)$ between the Hesse model $E_{K 3}$ and the Weierstrass model $E_{1}$.

\section{On automorphisms of the K3 Surface $X_{\lambda}$}

5.1. On the Mordell-Weil lattice of the elliptic fibration $\varphi$. For a point $P \in E_{K 3}(\mathbb{Q}(\omega, t))$, let us denote by $(P) \hookrightarrow X_{\lambda}$ the corresponding section of $\varphi: X_{\lambda} \rightarrow \mathbb{P}^{1}$. We denote by $\tau \in \operatorname{Aut}\left(X_{\lambda}\right)$ the automorphism which is the translation by $A_{1}^{\prime}$. We have 
Theorem 27. Modulo torsion, the section $A_{1}^{\prime}=\left(P_{1}\right)$ generates the MordellWeil lattice $M W L\left(X_{\lambda}\right)$ of sections.

Remark 28. One can compute the classes in $\mathrm{NS}\left(X_{\lambda}\right)$ of the curves $\left(R_{i}\right)$ (which are the translate by $\left(-P_{1}\right)$ of the curves $\left.A_{i}\right)$; we give these classes in the Appendix. Using that knowledge, we get the matrix representation on $\operatorname{NS}\left(X_{\lambda}\right)$ of the action of the automorphism $\tau$. The characteristic polynomial of $\tau$ is $(T-1)^{3}\left(T^{2}+T+1\right)^{8}$.

Proof. Let $O=A_{1}$ be the zero section, and let $F$ be a fiber of $\varphi: X_{\lambda} \rightarrow \mathbb{P}^{1}$. Using the knowledge of the action of the automorphism $\tau$ (which is the translation by $\left.\left(P_{1}\right)\right)$ on $\mathrm{NS}\left(X_{\lambda}\right)$, we get that

$$
\left(6 P_{1}\right)-2\left(3 P_{1}\right)+O \equiv 6 F
$$

in $\operatorname{NS}\left(X_{\lambda}\right)$, thus (see e.g. [26, Chapter III, Theorem 9.5]) $\left\langle 3 P_{1}, 3 P_{1}\right\rangle=6$ and $\left\langle P_{1}, P_{1}\right\rangle=\frac{2}{3}$, where $\langle\cdot, \cdot\rangle$ is the bilinear pairing on $\operatorname{MWL}\left(X_{\lambda}\right)$ associated to the canonical height.

Let $\operatorname{Triv}\left(X_{\lambda}\right)$ be the lattice generated the zero section and the fibers components of the fibration. The determinant formula [25, Corollary 6.39] is

$$
\left|\operatorname{det} \operatorname{NS}\left(X_{\lambda}\right)\right|=\left|\operatorname{det} \operatorname{Triv}\left(X_{\lambda}\right)\right| \cdot \operatorname{det} \operatorname{MWL}\left(X_{\lambda}\right) /\left|\operatorname{MWL}\left(X_{\lambda}\right)\right|^{2} .
$$

By Lemma 8, we know that $\left|\operatorname{det} \operatorname{NS}\left(X_{\lambda}\right)\right|=54$. We have moreover $\operatorname{det} \operatorname{Triv}\left(X_{\lambda}\right)=$ $-3^{8}$ and $\left|\operatorname{MWL}\left(X_{\lambda}\right)\right|^{2}=3^{4}$, thus we obtain that

$$
\operatorname{det} \operatorname{MWL}\left(X_{\lambda}\right)=\frac{2}{3} \text {. }
$$

By Theorem 18, the group $\operatorname{MWL}\left(X_{\lambda}\right)$ has $\operatorname{rank} 1$; since $\left\langle P_{1}, P_{1}\right\rangle=\frac{2}{3}$, we conclude that $P_{1}$ generates $\operatorname{MWL}\left(X_{\lambda}\right)$ modulo torsion.

Using the action of $\tau$ and its powers, we can obtain more classes in $\operatorname{NS}\left(X_{\lambda}\right)$ of the sections on the K3 surface $X_{\lambda} \rightarrow \mathbb{P}^{1}$.

Remark 29. We searched the $9 \mathbf{A}_{2}$-configurations among a set of 45 sections, but we obtained only the expected ones, i.e. the $9 \mathbf{A}_{2}$-configuration that are translate of the configuration $A_{i}, A_{i}^{\prime}, i \in\{1, \ldots, 9\}$. Since these configurations are images of one configuration by an automorphism (the translation by $A_{1}^{\prime}$ and its multiples), these $9 \mathbf{A}_{2}$-configurations give the same generalized Kummer structure.

5.2. More elements of the automorphism group and another double plane model. The K3 surface $X_{\lambda}$ is constructed as the minimal desingularization of the double cover of the plane branched over the sextic curve $C_{\lambda}$. Let $\sigma \in \operatorname{Aut}\left(X_{\lambda}\right)$ be the corresponding involution. By construction $\sigma\left(A_{j}\right)=A_{j}^{\prime}, \sigma\left(A_{j}^{\prime}\right)=A_{j}$ and $\sigma$ preserves the fiber of the fibration $X_{\lambda} \rightarrow \mathbb{P}^{1}$. From these facts, we know the action of $\sigma$ on the Néron-Severi lattice, since we know also the action of $\tau$, and one can compute that

Lemma 30. We have $\sigma=\tau \sigma \tau$. 
Let us recall that we denoted by $\left(R_{i}\right)$ the sections corresponding to the points $R_{i}=-P_{1}+Q_{i}$ in $E_{K 3}$. We also have a model $X_{\lambda} \subset \mathbb{P}^{1} \times \mathbb{P}^{2}$. From the equations of the curves involved, we obtain that:

a) the natural map $\pi_{2}: X_{\lambda} \rightarrow \mathbb{P}^{2}$ induced by the projection $\mathbb{P}^{1} \times \mathbb{P}^{2} \rightarrow \mathbb{P}^{2}$ is a 2 to 1 map,

b) it contracts the $(-2)$-curves $A_{1}, \ldots, A_{9}$ to the 9 torsion base points $\mathcal{T}_{9}$ of the Hesse pencil $x^{3}+y^{3}+z^{3}-3 \mu x y z=0, \mu \in \mathbb{P}^{1}$.

c) for $j \in\{1, \ldots, 9\}$, the curves $A_{j}^{\prime}$ and $\left(R_{j}\right)$ are mapped to a line $L_{j}^{\prime}$ that contains exactly one point of $\mathcal{T}_{9}$, that line is therefore tangent to the sextic curve at its other intersection points. The 9 lines are in general position.

We can therefore compute the action of the involution $\sigma^{\prime} \in \operatorname{Aut}\left(X_{\lambda}\right)$ corresponding to the double cover $\pi_{2}$ on the Néron-Severi lattice (the curves $A_{j}^{\prime}$ and $\left(R_{j}\right)$ are exchanged and one can check that the fiber is preserved; the classes of $\left(R_{j}\right)$ in base $F, A_{j}, A_{j}^{\prime}$ are in the appendix). The action of $\sigma^{\prime}$ on $X_{\lambda} \hookrightarrow \mathbb{P}^{5}$ is given in sub-section 4.3.3. We get:

Lemma 31. We have $\tau=\sigma \sigma^{\prime}$.

We compute moreover that the pull-back by $\pi_{2}$ of the 9 points of $\mathcal{T}_{9}$ are the irreducible curves $A_{1}, \ldots, A_{9}$, in fact we have

Theorem 32. The surface $X_{\lambda}$ is the minimal desingularization of the double cover of the plane branched over the sextic curve $C_{6}$ which is the union of the elliptic curves

$$
E_{\lambda}: x^{3}+y^{3}+z^{3}-3 \lambda x y z=0
$$

and the Hessian of $E_{\lambda}$ :

$$
\operatorname{He}(\lambda): x^{3}+y^{3}+z^{3}+\frac{\left(\lambda^{3}-4\right)}{\lambda^{2}} x y z=0 .
$$

Proof. Let $F_{0}$ and $F_{\infty}$ be the two fibers of the fibration $X_{\lambda} \rightarrow \mathbb{P}^{1}$ at 0 and infinity. One computes that $\pi_{2}\left(F_{0}\right)=E_{\lambda}, \pi_{2}\left(F_{\infty}\right)=\mathrm{He}(\lambda)$, and moreover

$$
\pi_{2}^{*}\left(E_{\lambda}\right)=2 F_{0}+\sum_{j=1}^{9} A_{j}, \pi_{2}^{*}(\operatorname{He}(\lambda))=2 F_{\infty}+\sum_{j=1}^{9} A_{j},
$$

thus $E_{\lambda}+\operatorname{He}(\lambda)$ is the branch locus of the map $\pi_{2}$. By Bézout Theorem, the singularities of $E_{\lambda}+\operatorname{He}(\lambda)$ are nodal since the curves $E_{\lambda}$ and $\operatorname{He}(\lambda)$ meet at $\mathcal{T}_{9}$

Remark 33. For each $j=1, \ldots, 9$, the images by $\pi_{2}$ of the two sections $\left(-2 P_{1}+Q_{j}\right),\left(2 P_{1}+Q_{j}\right)$ is the same quartic curve, which is nodal with 3 nodes. The images of $\left(-P_{1}+Q_{j}\right),\left(P_{1}+Q_{j}\right)$ are the 9 lines, their coordinates in the dual plane with basis $x, y, z$ are the same as the points in $\mathcal{P}_{9}$. These 9 lines are the inflection lines of the curve $E_{\lambda}$.

We recall that the Hesse configuration is the point-line configuration $\left(9_{4}, 12_{3}\right)$ of the 9 points in $\mathcal{T}_{9}$ and the 12 lines $\mathcal{L}_{12}$ such that each line contains 3 points in $\mathcal{T}_{9}$ and each point is on 4 lines. 
Proposition 34. The images by $\pi_{2}$ in $\mathbb{P}^{2}$ of the 24 irreducible components of the singular fibers of the fibration $\varphi: X_{\lambda} \rightarrow \mathbb{P}^{1}$ are the 12 lines of the Hesse configuration.

Proof. We give in Theorem 26 the 8 points $p \in \mathbb{P}^{1}$ such that the fiber $F_{p}$ over $p$ is singular. We are then able to compute these singular fibers in $X_{\lambda} \subset \mathbb{P}^{1} \times \mathbb{P}^{2}$ and their images in $\mathbb{P}^{2}$.

Remark 35 . Using the elliptic curve $E_{K 3}$, we obtain that the sub-group Tor $_{3}$ of order 3 elements in the Mordell-Weil lattice $\operatorname{MWL}\left(X_{\lambda}\right)$ is generated by two order 3 elements $t_{1}, t_{2}$ which acts on $\operatorname{NS}\left(X_{\lambda}\right)$ via

$$
t_{1}\left(A_{j}\right)=A_{\sigma j}, t_{1}\left(A_{j}^{\prime}\right)=A_{\sigma j}^{\prime}, \quad t_{2}\left(A_{j}\right)=A_{\mu j}, t_{2}\left(A_{j}^{\prime}\right)=A_{\mu j}^{\prime}
$$

where $\sigma=(1,2,3)(4,5,6)(7,8,9)$ and $\mu=(1,4,7)(2,5,8)(3,6,9)$. The elements of $\mathrm{Tor}_{3}$ commute with $\sigma$ (and of course with $\tau$ ). The action of $\mathrm{Tor}_{3}$ is transitive on the nine $9 \mathbf{A}_{2}$-configurations we found in section 3.2 .

5.3. On the stabilizer group of natural $9 \mathbf{A}_{2}$-configuration. Recall that $X_{\lambda}$ is the minimal desingularization of the double cover of $\mathbb{P}^{2}$ ramified over the 9-cuspidal sextic $C_{\lambda}$. The strict transform on $X_{\lambda}$ of $C_{\lambda}$ is a smooth elliptic curve isomorphic to $E_{\lambda}$. The linear system defined by that elliptic curve defines an elliptic fibration which we denote $\varphi: X_{\lambda} \rightarrow \mathbb{P}^{1}$, and which we called the natural fibration. The curves $A_{j}, A_{j}^{\prime}$ (above the cusps) are sections of $\varphi$, the $24(-2)$-curves $\theta_{J}, \theta_{J}^{\prime}$ (for some set $J \subset\{1, \ldots, 9\}$ of order 6 ) above the 12 conics are contained in the fibers (see Proposition 18), their classes are given in the Appendix, under a simpler labelling $\Theta_{j}, j \in\{1, \ldots, 24\}$. We have

Proposition 36. An integral basis $\mathcal{B}$ of the Néron-Severi lattice of $X_{\lambda}$ is

$$
\begin{gathered}
F, A_{1}, A_{1}^{\prime}, A_{2}, A_{2}^{\prime}, A_{3}, A_{3}^{\prime}, A_{4}, A_{4}^{\prime}, A_{5}, A_{5}^{\prime}, A_{6}, A_{7}, A_{7}^{\prime}, \\
\Theta_{5}, \Theta_{14}, \Theta_{22}, \Theta_{23}, \Theta_{20},
\end{gathered}
$$

where $F$ is a fiber of the fibration $\varphi$. The discriminant group $A_{\mathrm{NS}\left(X_{\lambda}\right)} \simeq$ $\mathbb{Z} / 2 \mathbb{Z} \times(\mathbb{Z} / 3 \mathbb{Z})^{3}$ is generated by

$$
\begin{aligned}
& w_{0}=\frac{1}{2}(0,1,1,0,0,0,0,1,1,0,0,0,1,1,1,1,1,0,0)_{\mathcal{B}}, \\
& v_{1}^{\prime}=\frac{1}{3}\left(A_{3}+2 A_{3}^{\prime}+A_{5}+2 A_{5}^{\prime}+A_{7}+2 A_{7}^{\prime}\right), \\
& v_{2}^{\prime}=\frac{1}{3}\left(A_{2}+2 A_{2}^{\prime}+2 A_{4}+A_{4}^{\prime}+2 A_{5}+A_{5}^{\prime}+A_{7}+2 A_{7}^{\prime}\right), \\
& v_{3}^{\prime}=\frac{1}{3}\left(A_{1}+2 A_{1}^{\prime}+A_{4}+2 A_{5}^{\prime}+A_{7}+2 A_{7}^{\prime}\right) .
\end{aligned}
$$

Proof. By Lemma 8, the discriminant group of the Néron-Severi lattice has order 54. The lattice generated by the elements in $\mathcal{B}$ has rank 19 and discriminant equal to 54, thus these elements generate $\operatorname{NS}\left(X_{\lambda}\right)$. By taking the inverse of the intersection matrix of the vectors in $\mathcal{B}$, we get the generators of the discriminant group.

The non-immediate part of the Gram matrix of the basis $\mathcal{B}$ is the intersection matrix of the 5 curves $\Theta_{j}, j=5,14,22,23,20$ with the curves in $\mathcal{B}$, whose matrix is: 


$$
\left(\begin{array}{ccccccccccccccccccc}
0 & 1 & 0 & 0 & 1 & 0 & 0 & 1 & 0 & 0 & 1 & 0 & 1 & 0 & -2 & 1 & 0 & 0 & 0 \\
0 & 0 & 1 & 0 & 0 & 1 & 0 & 0 & 1 & 0 & 0 & 1 & 0 & 1 & 1 & -2 & 0 & 0 & 0 \\
0 & 0 & 0 & 0 & 1 & 1 & 0 & 0 & 0 & 0 & 1 & 1 & 0 & 0 & 0 & 0 & -2 & 0 & 0 \\
0 & 0 & 0 & 0 & 0 & 0 & 0 & 0 & 1 & 0 & 1 & 0 & 1 & 0 & 0 & 0 & 0 & -2 & 0 \\
0 & 0 & 0 & 0 & 1 & 1 & 0 & 1 & 0 & 0 & 0 & 0 & 0 & 1 & 0 & 0 & 0 & 0 & -2
\end{array}\right)
$$

Let $G_{\mathcal{C}}$ be the automorphism sub-group of Aut $\left(X_{\lambda}\right)$ preserving globally the configuration $\mathcal{C}=A_{1}, A_{1}^{\prime}, \ldots, A_{9}, A_{9}^{\prime}$. The proof of the following Proposition will also serve as a preliminary for the proof of Theorem 39 in the next section:

Proposition 37. The group $G_{\mathcal{C}}$ is isomorphic to $\mathbb{Z}_{2} \times\left(\mathbb{Z}_{3} \rtimes S_{3}\right)$, where $\mathbb{Z}_{3} \rtimes S_{3}$ acts symplectically on $X_{\lambda}$. The center of $G_{\mathcal{C}}$ is generated by the non-symplectic involution $\sigma$ associated to the double cover map $X_{\lambda} \rightarrow \mathbb{P}^{2}$ branched over the cuspidal sextic curve $C_{\lambda}$.

Proof. Let $\phi$ be an element of $G_{\mathcal{C}}$. Since it preserves globally the configuration $\mathcal{C}$, it must map its orthogonal complement (generated by $D_{2}$, the pull-back of a line) to itself. There are $2^{9} 9$ ! bijective maps

$$
\mu:\left\{A_{1}, A_{1}^{\prime}, \ldots, A_{9}, A_{9}^{\prime}\right\} \rightarrow\left\{A_{1}, A_{1}^{\prime}, \ldots, A_{9}, A_{9}^{\prime}\right\}
$$

which preserves the incidence relations of $\mathcal{C}$. Since a linear map is defined by the images of the vectors of a basis, each map $\mu$ extends to a linear automorphism $\phi_{\mu}: \mathrm{NS}\left(X_{\lambda}\right) \otimes \mathbb{Q} \rightarrow \mathrm{NS}\left(X_{\lambda}\right) \otimes \mathbb{Q}$ sending $D_{2}$ to $D_{2}$ and the configuration $\mathcal{C}$ to itself. The action of $\phi$ on $\mathrm{NS}\left(X_{\lambda}\right)$ must be one of these maps. Using the integral basis $\mathcal{B}$ of Proposition 36, we obtain that among all the possibilities, only 864 matrices in basis $\mathcal{B}$ of such $\phi_{\mu}$ are in $G L_{19}(\mathbb{Z})$. These 864 matrices are the elements of a group $G_{864}$ isomorphic to the product of $\mathbb{Z} / 2 \mathbb{Z}$ with $A G L_{2}\left(\mathbb{F}_{3}\right)$, the affine linear group of the space $\mathbb{F}_{3}^{2}$. The center of $G_{864}$ has order 2 and is generated by the matrix of the non-symplectic involution $\sigma$ defined in section 5.2. In the appendix, we give two generators $g_{1}, g_{2}$ (of respective order 8 and 6) of the group $G_{432} \subset$ $G_{864}$ isomorphic to $A G L_{2}\left(\mathbb{F}_{3}\right)$. Their action on the 2 -torsion part of the discriminant group $A_{\mathrm{NS}\left(X_{\lambda}\right)}$ is trivial, and one computes that their action on the 3 -torsion part of the discriminant group $A_{\mathrm{NS}\left(X_{\lambda}\right)}$ is by the matrices

$$
\bar{g}_{1}=\left(\begin{array}{lll}
1 & 1 & 1 \\
2 & 1 & 0 \\
1 & 0 & 0
\end{array}\right), \bar{g}_{2}=\left(\begin{array}{ccc}
1 & 0 & 0 \\
0 & 2 & 0 \\
0 & 0 & 1
\end{array}\right)
$$

in basis $v_{1}, v_{2}, v_{3}$. The group $G_{\text {Disc }}$ generated by $\bar{g}_{1}, \bar{g}_{2}$ is isomorphic to the symmetric group $S_{4}$. The kernel of the map $G_{432} \rightarrow G_{\text {Disc }}$ is a group $G_{18}$ isomorphic to $\mathbb{Z}_{3} \rtimes S_{3}$. Let $\operatorname{Tran}\left(X_{\lambda}\right)$ be the orthogonal complement of $\operatorname{NS}\left(X_{\lambda}\right)$ in $H^{2}\left(X_{\lambda}, \mathbb{Z}\right)$; it is a rank 3 signature $(2,1)$ lattice. There exists an isomorphism

$$
\gamma: A_{\operatorname{Tran}\left(X_{\lambda}\right)} \rightarrow A_{\mathrm{NS}\left(X_{\lambda}\right)}
$$

between the discriminant groups, such that the quadratic forms of the groups satisfy $q_{\operatorname{Tran}\left(X_{\lambda}\right)} \circ \gamma=-q_{\mathrm{NS}\left(X_{\lambda}\right)}$. Since an element $\phi \in G_{18}$ acts trivially on $A_{\mathrm{NS}\left(X_{\lambda}\right)}$, we can extend it to an isometry $\Gamma$ of $H^{2}\left(X_{\lambda}, \mathbb{Z}\right)$ by gluing it with 
the identity map on $\operatorname{Tran}\left(X_{\lambda}\right)$ (see e.g. [30, Theorem 12] and references therein for an example of such construction). Since $\Gamma$ is the identity on the space $\operatorname{Tran}\left(X_{\lambda}\right) \otimes \mathbb{C}$ containing the period, this is a Hodge isometry. Since $\Gamma$ naturally preserves the polarization $D_{14}=4 D_{2}-\sum_{j}\left(A_{j}+A_{j}^{\prime}\right)$ (studied in Proposition (19), it is effective. Therefore we can apply the Torelli Theorem for K3 surfaces (see [6, Chap. VIII, Theorem 11.1]) and conclude that there exists a unique $g \in \operatorname{Aut}\left(X_{\lambda}\right)$ such that $g^{*}=\Gamma$ on $\operatorname{NS}\left(X_{\lambda}\right)$.

By [14, Corollary 3.3.5], since the Picard number of $X_{\lambda}$ is odd, the only Hodge isometry on $\operatorname{Tran}\left(X_{\lambda}\right)$ is $\pm I_{d}$. Suppose that an element $g$ of $G_{432}$ not contained in $G_{18}$ comes from an automorphism of $X_{\lambda}$. From the definition of $g$, its action $\bar{g}$ on $A_{\mathrm{NS}\left(X_{\lambda}\right)}$ is non trivial, thus its action on $\operatorname{Tran}\left(X_{\lambda}\right)$ is also non-trivial. The automorphism $g$ is therefore non symplectic and it acts by $-I d$ on $\operatorname{Tran}\left(X_{\lambda}\right)$. Thus $\bar{g} \in G_{\text {Disc }}$ acts on the discriminant group $A_{\operatorname{Tran}\left(X_{\lambda}\right)}$ by $-I d$. However $-I d$ is not contained in $G_{\text {Disc }} \simeq S_{4}$ : a contradiction and $g$ cannot come from an automorphism of $X_{\lambda}$.

Since $\sigma$ commutes with the elements of $G_{432}$, the automorphism group of the configuration is the direct product of $G_{18}$ and $\langle\sigma\rangle \simeq \mathbb{Z}_{2}$.

Remark 38. We give a geometric interpretation of the some elements of the group $G_{\mathcal{C}} \simeq \mathbb{Z}_{2} \times\left(\mathbb{Z}_{3} \rtimes S_{3}\right)$ in sub-section 4.3.3, the group $G_{\mathcal{C}}$ contains the 9 translations by 3 -torsion automorphisms (from the fibration $\varphi: X_{\lambda} \rightarrow \mathbb{P}^{1}$ ), and the involution $\sigma$ from the double cover $X_{\lambda} \rightarrow \mathbb{P}^{2}$. One can recover the automorphisms in the subgroup $\mathbb{Z}_{3} \rtimes S_{3}$ of $G_{\mathcal{C}}$ as follows:

Let $A$ be the abelian surface and let $J_{A}$ be the order 3 symplectic automorphism acting on $A$ such that $X_{\lambda}=\mathrm{Km}_{3}(A)$ is the minimal resolution of $A / J_{A}$, the exceptional locus being $\mathcal{C}$. The automorphism $J_{A}$ fixes 9 points $0=s_{1}, \ldots, s_{9}$ which form a group isomorphic to $\left(\mathbb{Z}_{3}\right)^{2}$. The group generated by the translation by the $s_{k}$ and the involution [-1] : $z \in A \rightarrow-z \in A$ is isomorphic to $\mathbb{Z}_{3} \rtimes S_{3}$. These isomorphisms induce automorphisms on $A / J_{A}$, hence automorphisms on K3 surface $X_{\lambda}$; these automorphisms preserve $\mathcal{C}$.

5.4. An automorphism sending a $9 \mathbf{A}_{2}$-configuration to another. Let

$$
\mathcal{C}^{\prime}=B_{1}, B_{1}^{\prime}, \ldots, B_{9}, B_{9}^{\prime}
$$

be one of the nine $9 \mathbf{A}_{2}$-configurations found in section 3.2 (so that $B_{j} B_{j}^{\prime}=1$, $B_{j} B_{k}=0$ for $j \neq k$ ), namely we choose the $9 \mathbf{A}_{2}$-configuration

$\mathcal{C}^{\prime}=\Theta_{12}, \Theta_{9}, \Theta_{14}, \Theta_{5}, \Theta_{16}, \Theta_{7}, \Theta_{2}, \Theta_{3}, \gamma_{1}, \gamma_{1}^{\prime}, \Theta_{4}, \Theta_{1}, \Theta_{8}, \Theta_{15}, \Theta_{6}, \Theta_{13}, \Theta_{10}, \Theta_{11}$,

where the classes of the $(-2)$-curves $\Theta_{j}$ (which are the curves $\theta_{i, j, k, m, n, o}$ more conveniently labelled and numbered) and $\gamma_{1}, \gamma_{1}^{\prime}$ are in the Appendix. The eight $\mathbf{A}_{2}$-configurations $B_{j}, B_{j}^{\prime}, j \in\{1, \ldots, 4,6, \ldots, 9\}$, are contained in the fibers of $\varphi$ and the $\mathbf{A}_{2}$-configuration $B_{5}, B_{5}^{\prime}$ is the strict transform under the double cover map of a the quartic curve $Q_{1}$.

Theorem 39. There exists an automorphism $f$ of $X_{\lambda}$ sending the curve $A_{j}$ (resp. $A_{j}^{\prime}$ ) to the curve $B_{j}$ (resp. $\left.B_{j}^{\prime}\right)$ for $j \in\{1, \ldots, 9\}$. In particular, the 
configuration $\mathcal{C}=A_{1}, A_{1}^{\prime}, \ldots, A_{9}, A_{9}^{\prime}$ is sent by $f$ to the configuration $\mathcal{C}^{\prime}$, and therefore the configuration $\mathcal{C}^{\prime}$ is on the $\operatorname{Aut}\left(X_{\lambda}\right)$-orbit of $\mathcal{C}$.

Proof. For finding the automorphism $f$ we proceeded as in Proposition 37. Let $\phi \in \operatorname{Aut}\left(X_{\lambda}\right)$ be an automorphism sending $\mathcal{C}$ to $\mathcal{C}^{\prime}$. The orthogonal complement of $\mathcal{C}$ is generated by $D_{2}$, the pull-back of a line in $\mathbb{P}^{2}$ and the orthogonal complements of $\mathcal{C}^{\prime}$ is generated by the divisor

$$
D_{2}^{\prime}=7 D_{2}-2 \sum_{j=1}^{j=9}\left(A_{j}+A_{j}^{\prime}\right)-2\left(A_{1}+A_{1}^{\prime}\right),
$$

of square 2. Since $\phi$ must preserve the Néron-Severi lattice, we have $\phi\left(D_{2}\right)=$ $D_{2}^{\prime}$. There are $2^{9} 9$ ! bijective maps

$$
\mu:\left\{A_{1}, A_{1}^{\prime}, \ldots, A_{9}, A_{9}^{\prime}\right\} \rightarrow\left\{B_{1}, B_{1}^{\prime}, \ldots, B_{9}, B_{9}^{\prime}\right\}
$$

which preserves the incidence relations of $\mathcal{C}$ and $\mathcal{C}^{\prime}$ and which extend uniquely to isometries $\tilde{\phi}_{\mu}$ of $\operatorname{NS}\left(X_{\lambda}\right) \otimes \mathbb{Q}$. If two of these maps $\tilde{\phi}_{1}, \tilde{\phi}_{2}$ preserve the lattice $\mathrm{NS}\left(X_{\lambda}\right)$, then $\tilde{\phi}_{2}^{-1} \tilde{\phi}_{1}$ also preserves that lattice, moreover it also preserves the configuration $\mathcal{C}$. It is therefore an element of the group $G_{864}$ defined in the proof of Proposition 37. Thus the set of maps $\tilde{\phi}$ (among the maps $\left.\tilde{\phi}_{\mu}\right)$ that preserve $\operatorname{NS}\left(X_{\lambda}\right)$ is the orbit of $\tilde{\phi}_{1}$ under the action of $G_{864}$ on the left. The element $\tilde{\phi}_{1}$ defined by sending $A_{j}$ to $B_{j}$ and $A_{j}^{\prime}$ to $B_{j}^{\prime}$ preserves the lattice $\mathrm{NS}\left(X_{\lambda}\right)$ (its matrix $f_{\mathcal{B}}$ in basis $\mathcal{B}$ is given in the Appendix). Its action on the discriminant group is trivial. Then, as in the proof of Proposition 37, we can extend $\tilde{\phi}_{1}$ to an Hodge isometry of $H^{2}\left(X_{\lambda}, \mathbb{Z}\right)$.

Let us prove that this isometry is effective. The polarization (see Proposition 19l) $D_{14}=4 D_{2}-\sum\left(A_{j}+A_{j}^{\prime}\right)$ is sent to $D_{14}^{\prime}=4 D_{2}^{\prime}-\sum\left(B_{j}+B_{j}^{\prime}\right)=$ $D_{2}^{\prime}+F_{1}$ where $F_{1}$ is (the class of) a fiber of a fibration $f_{1}$ by Remark 40. Using the classes of the curves in $\operatorname{NS}\left(X_{\lambda}\right)$, it is easy to check that the curves $B_{j}, B_{j}^{\prime}$ are 18 sections of $f_{1}$.

The degree 7 curve $Q \hookrightarrow \mathbb{P}^{2}$ defined by

$$
\begin{gathered}
\lambda x^{7}-2 \lambda^{2} x^{6} y+3 \lambda^{3} x^{5} y^{2}-\left(2 \lambda^{4}+\lambda\right) x^{4} y^{3}+\lambda^{5} x^{3} y^{4}+3 \lambda^{3} x^{2} y^{5} \\
+\lambda^{2} y^{7}-2 \lambda^{2} x^{6} z-1 x^{5} y z+4 \lambda x^{4} y^{2} z-\left(2 \lambda^{5}+9 \lambda^{2}\right) x^{3} y^{3} z+\left(\lambda^{3}+1\right) x^{2} \\
y^{4} z-\left(3 \lambda^{4}+2 \lambda\right) x y^{5} z-2 \lambda^{2} y^{6} z+3 \lambda^{3} x^{5} z^{2}+4 \lambda x^{4} y z^{2}+\left(3 \lambda^{5}+4 \lambda^{2}\right) x^{3} y^{2} z^{2} \\
+\left(4 \lambda^{3}-1\right) x^{2} y^{3} z^{2}+4 \lambda x y^{4} z^{2}+\lambda^{2} y^{5} z^{2}-\left(2 \lambda^{4}+\lambda\right) x^{4} z^{3}-\left(2 \lambda^{5}+9 \lambda^{2}\right) x^{3} y z^{3} \\
+\left(4 \lambda^{3}-1\right) x^{2} y^{2} z^{3}-\left(3 \lambda^{4}+4 \lambda\right) x y^{3} z^{3}+\lambda^{5} y^{4} z^{3}+\lambda^{5} x^{3} z^{4}+\left(\lambda^{3}+1\right) x^{2} y z^{4} \\
+4 \lambda x y^{2} z^{4}+\lambda^{5} y^{3} z^{4}+3 \lambda^{3} x^{2} z^{5}-\left(3 \lambda^{4}+2 \lambda\right) x y z^{5}+\lambda^{2} y^{2} z^{5}-2 \lambda^{2} y z^{6}+\lambda^{2} z^{7}=0
\end{gathered}
$$

is irreducible with singularities of multiplicity 4 at the point $p_{1}$ in $\mathcal{P}_{9}$, and with multiplicity 2 at the eight remaining points (the curve $Q$ was found by using LinSys). The geometric genus of $Q$ is 1 and $Q$ meets the branch locus at two other points, so that its strict transform on $X_{\lambda}$ is smooth of genus 2 in the linear system $\left|D_{2}^{\prime}\right|$. Thus $D_{2}^{\prime}$ is effective, nef and $\left|D_{2}^{\prime}\right|$ is base point free.

Let $C$ be an irreducible $(-2)$-curve on $X_{\lambda}$. If $C F^{\prime}=C D_{2}^{\prime}=0$, then $C$ is contained in a fiber of $f_{1}$ and is contracted by $\left|D_{2}^{\prime}\right|$. The second point implies that $C$ is one of the curves $B_{j}, B_{j}^{\prime}$ (otherwise the Picard number of 
$X_{\lambda}$ would be $>19$ ), but then one has $C F^{\prime}=1$, which is a contradiction. Thus $D_{14}^{\prime}$ is ample and the isometry is effective.

We then conclude as in the proof of Proposition 37 that there exists an automorphism of $X_{\lambda}$ such that its action on the curves $A_{j}, A_{j}^{\prime}$ is as described.

We recall that for $k=1, \ldots, 9$, the point $p_{k} \in \mathcal{P}_{9}$ is the point over which $A_{k}+A_{k}^{\prime}$ is contracted by the double cover map $\eta: X_{\lambda} \rightarrow \mathbb{P}^{2}$.

Remark 40. For any point $p_{k}$, the pencil of lines through $p_{k}$ induces an elliptic fibration $f_{k}: X_{\lambda} \rightarrow \mathbb{P}^{1}$ such that the curves $A_{k}, A_{k}^{\prime}$ are sections and the curves $A_{j}, A_{j}^{\prime}$ for $j \neq k$ are contained in the fibers. The singular fibers of $f_{k}$ are also $8 \mathbf{A}_{2}$; from the known intersection numbers of these fibers with the elements of basis $D_{2}, A_{1}, \ldots, A_{9}^{\prime}$, we obtain that the class of a fiber is

$F_{k}=D_{2}-\left(A_{k}+A_{k}^{\prime}\right)=\frac{1}{3}\left(F+\sum_{j=1}^{9}\left(A_{j}+A_{j}^{\prime}\right)\right)-\left(A_{k}+A_{k}^{\prime}\right)=3 D_{2}^{\prime}-\sum_{j=1}^{9}\left(B_{j}+B_{j}^{\prime}\right)$ where $F$ is a fiber of $\varphi$ and the $B_{j}, B_{j}^{\prime}$ are the curve in the $9 \mathbf{A}_{9}$-configuration found in Section [3.2, $D_{2}^{\prime}$ being the generator of the orthogonal complement of the $B_{j}, B_{j}^{\prime}$ 's. The curves $B_{j}, B_{j}^{\prime}$ are sections of $f_{k}$.

So the geometric situation for the configuration $A_{1}, A_{1}^{\prime}, \ldots, A_{9}, A_{9}^{\prime}$ and fibration $f_{k}$ is very similar to the situation for $\mathcal{C}^{\prime}$ and fibration $\varphi$ for which 8 of the $\mathbf{A}_{2}$-configurations in $B_{1}, B_{1}^{\prime}, \ldots, B_{9}, B_{9}^{\prime}$ are in the 8 singular fibers and the remaining one are sections.

5.4.1. Aligned singularities of the union of the 12 conics. The fibration $\varphi$ has the remarkable property that the 18 sections $A_{j}, A_{j}^{\prime}$ meet on the same fiber, which is isomorphic to $E_{\lambda}$. It can be instructive to understand how the similar result holds for the fibration $f_{1}$ (see Remark 40) and the curves $B_{j}, B_{j}^{\prime}$, this is the aim of this subsection, which also gives an explanation why each line of the dual Hesse configuration contains 4 double points of the curve $\sum_{C \in \mathcal{C}_{12}} C$ and these double points form the set $\mathcal{P}_{12}$.

We recall that the 12 conics in $\mathcal{C}_{12}$ meet in either 4 or 3 points in $\mathcal{P}_{9}$. If two conics meet in 3 points in $\mathcal{P}_{9}$ then the fourth intersection point is an ordinary singularity of the union of the 12 conics. Above the 8 conics that contain $p_{1}$ are the $16(-2)$-curves that gives a $8 \mathbf{A}_{8}$-configuration, which one can complete to a $9 \mathbf{A}_{2}$-configuration according to section 3.2 .

The 8 conics containing $p_{1}$ have the property that they meet by pairs into 4 points $q_{1}, \ldots, q_{4}$ not in $\mathcal{P}_{9}$ and that these 4 points are on a line containing $p_{1}$. That line $L_{1}$ is the tangent line to the cusp $p_{1}$ (it is one of the lines of the dual Hesse configuration defined in Section 2.4). It meets the cuspidal sextic in $p_{1}$ (with multiplicity 3 ) and at points $r_{1}, r_{2}, r_{3}$. One can check that the fiber $F_{0}$ of $f_{1}$ which is the strict transform on $X_{\lambda}$ of $L_{1}$ is isomorphic to $E_{\lambda}$ (since we know the branch locus $F_{0} \rightarrow L_{1}$ ).

The 8 points in $X_{\lambda}$ above $q_{1}, \ldots, q_{4}$ are the meeting points of the curves in the $8 \mathbf{A}_{2}$ configuration obtained by taking the strict transform of the 8 conics trough $p_{1}$. Following the Figure 3.2, the $9^{\text {th }} \mathbf{A}_{2}$-configuration also has 
its intersection point on that fiber $F_{0}$. In fact, the fiber $F_{0}$ is the image by an automorphism of $X_{\lambda}$ of the fiber over 0 of $\varphi$.

The union of the dual Hesse configuration and the 12 conics in $\mathcal{C}_{12}$ is a lineconic arrangement with 9 points of multiplicity 9,12 points of multiplicity 5 and 72 double points, and with other remarkable properties studied in [19]. 


\section{APPENDix}

Let $D_{2}$ be the pull-back of a line by the double cover map $\eta: X_{\lambda} \rightarrow \mathbb{P}^{1}$. Let us define the following classes in the $\mathbb{Q}$-basis $\mathcal{B}_{0}=\left(D_{2}, A_{1}, A_{1}^{\prime}, \ldots, A_{9}, A_{9}^{\prime}\right)$ :

$$
B_{1}=2 D_{2}-\frac{1}{3}\left(\sum_{j=1}^{9} 2 A_{j}+A_{j}^{\prime}\right), B_{2}=2 D_{2}-\frac{1}{3}\left(\sum_{j=1}^{9} A_{j}+2 A_{j}^{\prime}\right) .
$$

We remark that $B_{1}^{2}=B_{2}^{2}=2, B_{1} B_{2}=5$ and $B_{1}+B_{2}=D_{14}$. We have $D_{14} A_{j}=D_{14} A_{j}^{\prime}=1$, therefore $B_{i} A_{j} \in\{0,1\}, B_{i} A_{j}^{\prime} \in\{0,1\}$. Using algorithms described in [20], we find that for $j \in\{1, \ldots, 9\}$, the classes of the curves $\gamma_{j}, \gamma_{j}^{\prime}$ above the quartic $Q_{j}$ are

$$
\gamma_{j}=B_{1}-\left(A_{j}+A_{j}^{\prime}\right), \gamma_{j}^{\prime}=B_{2}-\left(A_{j}+A_{j}^{\prime}\right) .
$$

It is easy to check that $\gamma_{j}^{2}=\gamma_{j}^{\prime 2}=-2, \gamma_{j} \gamma_{j}^{\prime}=1$, and for $1 \leq i \neq j \leq 9$, we have $\gamma_{i} \gamma_{j}=\gamma_{i}^{\prime} \gamma_{j}^{\prime}=0$ and $\gamma_{i} \gamma_{j}^{\prime}=3$. In fact, using that the image in $\mathbb{P}^{2}$ of $\gamma_{j}, \gamma_{j}^{\prime}$ is a quartic curve that goes through the points in $\mathcal{P}_{9}$ with a multiplicity 3 at $p_{j}$, one gets

$$
4 D_{2} \equiv \gamma_{j}+\gamma_{j}^{\prime}+2\left(A_{j}+A_{j}^{\prime}\right)+\sum_{j=1}^{9}\left(A_{j}+A_{j}^{\prime}\right) .
$$

The classes in the $\mathbb{Q}$-basis $\mathcal{B}_{0}=\left(L, A_{1}, A_{1}^{\prime}, \ldots, A_{9}, A_{9}^{\prime}\right)$ of the $24(-2)$ curves $\theta_{i, \ldots, n}, \theta_{i, \ldots, n}^{\prime}$ above the 12 conics $C_{i, \ldots, n}$ in $\mathcal{C}_{12}$ are

$$
\begin{aligned}
& \theta_{123456}=\frac{1}{3}(3,-2,-1,-2,-1,-2,-1,-1,-2,-1,-2,-1,-2,0,0,0,0,0,0), \\
& \theta_{123456}^{\prime}=\frac{1}{3}(3,-1,-2,-1,-2,-1,-2,-2,-1,-2,-1,-2,-1,0,0,0,0,0,0), \\
& \theta_{123789}=\frac{1}{3}(3,-2,-1,-2,-1,-2,-1,0,0,0,0,0,0,-1,-2,-1,-2,-1,-2), \\
& \theta_{123789}^{\prime}=\frac{1}{3}(3,-1,-2,-1,-2,-1,-2,0,0,0,0,0,0,-2,-1,-2,-1,-2,-1), \\
& \theta_{124578}=\frac{1}{3}(3,-2,-1,-1,-2,0,0,-2,-1,-1,-2,0,0,-2,-1,-1,-2,0,0), \\
& \theta_{124578}^{\prime}=\frac{1}{3}(3,-1,-2,-2,-1,0,0,-1,-2,-2,-1,0,0,-1,-2,-2,-1,0,0), \\
& \theta_{124689}=\frac{1}{3}(3,-2,-1,-1,-2,0,0,-1,-2,0,0,-2,-1,0,0,-2,-1,-1,-2), \\
& \theta_{124689}^{\prime}=\frac{1}{3}(3,-1,-2,-2,-1,0,0,-2,-1,0,0,-1,-2,0,0,-1,-2,-2,-1), \\
& \theta_{125679}=\frac{1}{3}(3,-2,-1,-1,-2,0,0,0,0,-2,-1,-1,-2,-1,-2,0,0,-2,-1), \\
& \theta_{125679}^{\prime}=\frac{1}{3}(3,-1,-2,-2,-1,0,0,0,0,-1,-2,-2,-1,-2,-1,0,0,-1,-2), \\
& \theta_{134589}=\frac{1}{3}(3,-2,-1,0,0,-1,-2,-1,-2,-2,-1,0,0,0,0,-1,-2,-2,-1), \\
& \theta_{134589}^{\prime}=\frac{1}{3}(3,-1,-2,0,0,-2,-1,-2,-1,-1,-2,0,0,0,0,-2,-1,-1,-2), \\
& \theta_{134679}=\frac{1}{3}(3,-2,-1,0,0,-1,-2,-2,-1,0,0,-1,-2,-2,-1,0,0,-1,-2), \\
& \theta_{134679}^{\prime}=\frac{1}{3}(3,-1,-2,0,0,-2,-1,-1,-2,0,0,-2,-1,-1,-2,0,0,-2,-1), \\
& \theta_{135678}=\frac{1}{3}(3,-2,-1,0,0,-1,-2,0,0,-1,-2,-2,-1,-1,-2,-2,-1,0,0), \\
& \theta_{135678}^{\prime}=\frac{1}{3}(3,-1,-2,0,0,-2,-1,0,0,-2,-1,-1,-2,-2,-1,-1,-2,0,0), \\
& \theta_{234579}=\frac{1}{3}(3,0,0,-2,-1,-1,-2,-2,-1,-1,-2,0,0,-1,-2,0,0,-2,-1), \\
& \theta_{234579}^{\prime}=\frac{1}{3}(3,0,0,-1,-2,-2,-1,-1,-2,-2,-1,0,0,-2,-1,0,0,-1,-2), \\
& \theta_{234678}=\frac{1}{3}(3,0,0,-2,-1,-1,-2,-1,-2,0,0,-2,-1,-2,-1,-1,-2,0,0), \\
& \theta_{234678}^{\prime}=\frac{1}{3}(3,0,0,-1,-2,-2,-1,-2,-1,0,0,-1,-2,-1,-2,-2,-1,0,0), \\
& \theta_{235689}=\frac{1}{3}(3,0,0,-2,-1,-1,-2,0,0,-2,-1,-1,-2,0,0,-2,-1,-1,-2), \\
& \theta_{235689}^{\prime}=\frac{1}{3}(3,0,0,-1,-2,-2,-1,0,0,-1,-2,-2,-1,0,0,-1,-2,-2,-1), \\
& \theta_{456789}=\frac{1}{3}(3,0,0,0,0,0,0,-1,-2,-1,-2,-1,-2,-2,-1,-2,-1,-2,-1), \\
& \theta_{456789}^{\prime}=\frac{1}{3}(3,0,0,0,0,0,0,-2,-1,-2,-1,-2,-1,-1,-2,-1,-2,-1,-2) .
\end{aligned}
$$

We also denote by $\Theta_{j}, j=1, \ldots, 24$ these curves in the order of the above list.

For $k=1, \ldots, 9$, the quartic curves $Q_{k}$ through $\mathcal{P}_{9}$ that have a multiplicity 3 singular point at $p_{k}$ are: 


$$
\begin{aligned}
& Q_{1}: x^{4}-2 \lambda x^{3} y+3 \lambda^{2} x^{2} y^{2}-\left(\lambda^{3}+1\right) x y^{3}+\lambda y^{4}-2 \lambda x^{3} z+\left(-\lambda^{3}+1\right) x y^{2} z-2 \lambda y^{3} z \\
& +3 \lambda^{2} x^{2} z^{2}+\left(-\lambda^{3}+1\right) x y z^{2}+\left(\lambda^{4}+2 \lambda\right) y^{2} z^{2}-\left(\lambda^{3}+1\right) x z^{3}-2 \lambda y z^{3}+\lambda z^{4}=0, \\
& Q_{2}: x^{4}-\left(\lambda^{3}+1\right) / \lambda x^{3} y+3 \lambda x^{2} y^{2}-2 x y^{3}+1 / \lambda y^{4}-2 x^{3} z+\left(-\lambda^{3}+1\right) / \lambda x^{2} y z-2 y^{3} z \\
& +\left(\lambda^{3}+2\right) x^{2} z^{2}+\left(1-\lambda^{3}\right) / \lambda x y z^{2}+3 \lambda y^{2} z^{2}-2 x z^{3}-\left(\lambda^{3}+1\right) / \lambda y z^{3}+z^{4}=0, \\
& Q_{3}: x^{4}-2 x^{3} y+\left(\lambda^{3}+2\right) x^{2} y^{2}-2 x y^{3}+y^{4}-\left(\lambda^{3}+1\right) / \lambda x^{3} z+\left(1-\lambda^{3}\right) / \lambda x^{2} y z \\
& +\left(1-\lambda^{3}\right) / \lambda x y^{2} z-\left(\lambda^{3}+1\right) / \lambda y^{3} z+3 \lambda x^{2} z^{2}+3 \lambda y^{2} z^{2}-2 x z^{3}-2 y z^{3}+1 / \lambda z^{4}=0, \\
& Q_{4}: x^{4}+(2 \omega+2) \lambda x^{3} y+3 \omega \lambda^{2} x^{2} y^{2}-\left(\lambda^{3}+1\right) x y^{3}-(\omega+1) \lambda y^{4}-2 \omega \lambda x^{3} z \\
& -\left(\omega^{2} \lambda^{3}+\omega+1\right) x y^{2} z-2 \omega \lambda y^{3} z-(3 \omega+3) \lambda^{2} x^{2} z^{2}+\left(\omega-\omega \lambda^{3}\right) x y z^{2} \\
& +\left(\lambda^{4}+2 \lambda\right) y^{2} z^{2}-\left(\lambda^{3}+1\right) x z^{3}+(2 \omega+2) \lambda y z^{3}+\omega \lambda z^{4}=0, \\
& Q_{5}: x^{4}-\left(\omega^{2} \lambda^{3}+\omega^{2}\right) / \lambda x^{3} y+3 \omega \lambda x^{2} y^{2}-2 x y^{3}-(\omega+1) / \lambda y^{4}-2 \omega x^{3} z \\
& +\left(1-\lambda^{3}\right) / \lambda x^{2} y z-2 \omega y^{3} z-\left((\omega+1) \lambda^{3}+2 \omega+2\right) x^{2} z^{2}+\left(\omega-\omega \lambda^{3}\right) / \lambda x y z^{2} \\
& +3 \lambda y^{2} z^{2}-2 x z^{3}-\omega^{2}\left(\lambda^{3}+1\right) / \lambda y z^{3}+\omega z^{4}=0, \\
& Q_{6}: x^{4}+(2 \omega+2) x^{3} y+\left(\omega \lambda^{3}+2 \omega\right) x^{2} y^{2}-2 x y^{3}+\omega^{2} y^{4}-\left(\omega \lambda^{3}+\omega\right) / \lambda x^{3} z \\
& +\left(1-\lambda^{3}\right) / \lambda x^{2} y z-\left(\omega^{2} \lambda^{3}-\omega^{2}\right) / \lambda x y^{2} z-\left(\omega \lambda^{3}+\omega\right) / \lambda y^{3} z \\
& -(3 \omega+3) \lambda x^{2} z^{2}+3 \lambda y^{2} z^{2}-2 x z^{3}+(2 \omega+2) y z^{3}+\omega / \lambda z^{4}=0, \\
& Q_{7}: x^{4}-2 \omega \lambda x^{3} y-(3 \omega+3) \lambda^{2} x^{2} y^{2}-\left(\lambda^{3}+1\right) x y^{3}+\omega \lambda y^{4}+(2 \omega+2) \lambda x^{3} z \\
& +\left(\omega-\omega \lambda^{3}\right) x y^{2} z+(2 \omega+2) \lambda y^{3} z+3 \omega \lambda^{2} x^{2} z^{2}-\left(\omega^{2} \lambda^{3}-\omega^{2}\right) x y z^{2} \\
& +\left(\lambda^{4}+2 \lambda\right) y^{2} z^{2}-\left(\lambda^{3}+1\right) x z^{3}-2 \omega \lambda y z^{3}+\omega^{2} \lambda z^{4}=0, \\
& Q_{8}: x^{4}-\left(\omega \lambda^{3}+\omega\right) / \lambda x^{3} y-(3 \omega+3) \lambda x^{2} y^{2}-2 x y^{3}+\omega / \lambda y^{4}+(2 \omega+2) x^{3} z \\
& +\left(1-\lambda^{3}\right) / \lambda x^{2} y z+(2 \omega+2) y^{3} z+\left(\omega \lambda^{3}+2 \omega\right) x^{2} z^{2}-\left(\omega^{2} \lambda^{3}-\omega^{2}\right) / \lambda x y z^{2} \\
& +3 \lambda y^{2} z^{2}-2 x z^{3}-\left(\omega \lambda^{3}+\omega\right) / \lambda y z^{3}+\omega^{2} z^{4}=0, \\
& Q_{9}: x^{4}-2 \omega x^{3} y+\left(\omega^{2} \lambda^{3}-2 \omega-2\right) x^{2} y^{2}-2 x y^{3}+\omega y^{4}-\left(\omega^{2} \lambda^{3}+\omega^{2}\right) / \lambda x^{3} z \\
& +\left(1-\lambda^{3}\right) / \lambda x^{2} y z+\left(-\omega \lambda^{3}+\omega\right) / \lambda x y^{2} z-\left(\omega^{2} \lambda^{3}+\omega^{2}\right) / \lambda y^{3} z \\
& +3 \omega \lambda x^{2} z^{2}+3 \lambda y^{2} z^{2}-2 x z^{3}-2 \omega y z^{3}+\omega^{2} / \lambda z^{4}=0,
\end{aligned}
$$

where $\omega^{2}+\omega+1=0$.

The matrices in basis $\mathcal{B}$ of the generators of the group $G_{432} \simeq A G L_{2}\left(\mathbb{F}_{3}\right)$ preserving the natural $9 \mathbf{A}_{2}$-configuration $A_{1}, A_{1}^{\prime}, \ldots, A_{9}, A_{9}^{\prime}$ are

$$
g_{1}=\left(\begin{array}{ccccccccccccccccccc}
1 & 0 & 0 & 0 & 0 & 0 & 0 & 1 & -2 & 0 & 0 & 0 & 0 & 0 & 0 & 1 & 0 & 1 & 0 \\
0 & 0 & 0 & 0 & 0 & -1 & 1 & 0 & 0 & 0 & 0 & 0 & 0 & 0 & 1 & 0 & 0 & -1 & 0 \\
0 & 0 & 0 & 0 & 0 & 0 & 1 & 0 & 0 & 0 & 0 & 0 & 0 & 0 & 1 & 0 & 0 & 0 & 0 \\
0 & 1 & 0 & 0 & 0 & 1 & -1 & 1 & -1 & 0 & 0 & 0 & 0 & 0 & -1 & 0 & 0 & 1 & 0 \\
0 & 0 & 1 & 0 & 0 & 2 & -2 & 1 & 0 & 0 & 0 & 0 & 0 & 0 & -1 & -1 & 0 & 1 & 0 \\
0 & 0 & 0 & 0 & 0 & 4 & -2 & 1 & 0 & 1 & 0 & 0 & 0 & 0 & -1 & -1 & -1 & 2 & 0 \\
0 & 0 & 0 & 0 & 0 & 2 & -1 & 1 & -1 & 0 & 1 & 0 & 0 & 0 & -1 & 0 & -1 & 1 & 0 \\
0 & 0 & 0 & 0 & 0 & -1 & 1 & 0 & 1 & 0 & 0 & 0 & 1 & 0 & 0 & 0 & 1 & -1 & 0 \\
0 & 0 & 0 & 0 & 0 & -2 & 2 & -1 & 1 & 0 & 0 & 0 & 0 & 1 & 1 & 0 & 1 & -1 & 0 \\
0 & 0 & 0 & 1 & 0 & -1 & 0 & 0 & -1 & 0 & 0 & 0 & 0 & 0 & 0 & 1 & 0 & 0 & 0 \\
0 & 0 & 0 & 0 & 1 & -2 & 0 & -1 & 0 & 0 & 0 & 0 & 0 & 0 & 0 & 1 & 0 & -1 & 0 \\
0 & 0 & 0 & 0 & 0 & 2 & -1 & 0 & 0 & 0 & 0 & 1 & 0 & 0 & 0 & -1 & -1 & 1 & 0 \\
0 & 0 & 0 & 0 & 0 & -3 & 2 & -1 & 1 & 0 & 0 & 0 & 0 & 0 & 1 & 1 & 1 & -2 & 0 \\
0 & 0 & 0 & 0 & 0 & 0 & 1 & 0 & 1 & 0 & 0 & 0 & 0 & 0 & 0 & 0 & 0 & 0 & 0 \\
0 & 0 & 0 & 0 & 0 & -2 & 1 & -1 & 2 & 0 & 0 & 0 & 0 & 0 & 1 & 0 & 1 & -2 & 0 \\
0 & 0 & 0 & 0 & 0 & 1 & 1 & -1 & 2 & 0 & 0 & 0 & 0 & 0 & 1 & -1 & 0 & 0 & 0 \\
0 & 0 & 0 & 0 & 0 & 3 & -3 & 0 & 0 & 0 & 0 & 0 & 0 & 0 & -1 & -1 & -1 & 1 & 0 \\
0 & 0 & 0 & 0 & 0 & -4 & 2 & -2 & 1 & 0 & 0 & 0 & 0 & 0 & 1 & 1 & 1 & -2 & 1 \\
0 & 0 & 0 & 0 & 0 & 2 & -1 & 1 & 1 & 0 & 0 & 0 & 0 & 0 & -1 & -1 & 0 & 1 & 0
\end{array}\right)
$$




$$
g_{2}=\left(\begin{array}{ccccccccccccccccccc}
1 & 0 & 0 & 0 & 0 & 0 & 0 & 1 & -2 & 0 & 0 & 0 & 0 & 0 & 0 & 1 & 0 & 1 & 0 \\
0 & 0 & 0 & 0 & 0 & -1 & 1 & 0 & 0 & 1 & 0 & 0 & 0 & 2 & 0 & 0 & 1 & 0 & 0 \\
0 & 0 & 0 & 0 & 0 & 0 & 1 & 0 & 0 & 0 & 1 & 0 & 0 & 1 & 0 & 0 & 0 & 0 & 0 \\
0 & 0 & 0 & 0 & 0 & 1 & -1 & 1 & -1 & 0 & 0 & 0 & 0 & -1 & 0 & 0 & -1 & 0 & 0 \\
0 & 0 & 0 & 0 & 0 & 2 & -2 & 1 & 0 & 0 & 0 & 0 & 0 & -2 & 0 & 0 & -1 & 0 & 0 \\
0 & 1 & 0 & 0 & 0 & 4 & -2 & 1 & 0 & 0 & 0 & 0 & 0 & -4 & 0 & 0 & -2 & 1 & 0 \\
0 & 0 & 1 & 0 & 0 & 2 & -1 & 1 & -1 & 0 & 0 & 0 & 0 & -2 & 0 & 0 & -1 & 1 & 0 \\
0 & 0 & 0 & 1 & 0 & -1 & 1 & 0 & 1 & 0 & 0 & 0 & 0 & 1 & 0 & 0 & 1 & 0 & 0 \\
0 & 0 & 0 & 0 & 1 & -2 & 2 & -1 & 1 & 0 & 0 & 0 & 0 & 2 & 0 & 0 & 1 & 0 & 0 \\
0 & 0 & 0 & 0 & 0 & -1 & 0 & 0 & -1 & 0 & 0 & 1 & 0 & 1 & 0 & 0 & 0 & 0 & 0 \\
0 & 0 & 0 & 0 & 0 & -2 & 0 & -1 & 0 & 0 & 0 & 0 & 0 & 2 & 0 & 0 & 1 & -1 & 0 \\
0 & 0 & 0 & 0 & 0 & 2 & -1 & 0 & 0 & 0 & 0 & 0 & 1 & -2 & 0 & 0 & -1 & 0 & 0 \\
0 & 0 & 0 & 0 & 0 & -3 & 2 & -1 & 1 & 0 & 0 & 0 & 0 & 3 & 0 & 0 & 2 & -1 & 0 \\
0 & 0 & 0 & 0 & 0 & 0 & 1 & 0 & 1 & 0 & 0 & 0 & 0 & 0 & 0 & 0 & 0 & 0 & 0 \\
0 & 0 & 0 & 0 & 0 & -2 & 1 & -1 & 2 & 0 & 0 & 0 & 0 & 3 & 0 & -1 & 2 & -1 & 0 \\
0 & 0 & 0 & 0 & 0 & 1 & 1 & -1 & 2 & 0 & 0 & 0 & 0 & 0 & 1 & -1 & 0 & 0 & 0 \\
0 & 0 & 0 & 0 & 0 & 3 & -3 & 0 & 0 & 0 & 0 & 0 & 0 & -3 & 0 & 0 & -2 & 0 & 0 \\
0 & 0 & 0 & 0 & 0 & -4 & 2 & -2 & 1 & 0 & 0 & 0 & 0 & 3 & 0 & 0 & 2 & -1 & 1 \\
0 & 0 & 0 & 0 & 0 & 2 & -1 & 1 & 1 & 0 & 0 & 0 & 0 & -3 & 0 & 0 & -1 & 0 & 0
\end{array}\right),
$$

The automorphism $f$ of Theorem 39 acts on the Néron-Severi lattice by

$$
f_{\mathcal{B}}=\left(\begin{array}{ccccccccccccccccccc}
0 & 1 & 0 & 0 & 0 & 0 & 1 & 0 & 1 & 1 & 0 & 0 & 0 & 0 & 0 & 0 & 0 & 0 & 1 \\
0 & 1 & -1 & 0 & 0 & -1 & 0 & 0 & 0 & -1 & 0 & 1 & -1 & 0 & 0 & 0 & 0 & 0 & 0 \\
0 & 0 & 0 & 0 & 0 & -1 & 0 & 0 & 0 & -1 & 0 & 0 & -1 & 0 & 0 & 0 & 0 & 0 & 0 \\
0 & 0 & 0 & 0 & 0 & 1 & 0 & 0 & 0 & 1 & -1 & -1 & 0 & 0 & 0 & 0 & 0 & 1 & 0 \\
0 & -1 & 0 & 0 & 0 & 2 & -1 & 0 & 0 & 1 & -1 & -2 & 1 & 0 & 0 & 0 & 0 & 0 & -1 \\
0 & -3 & 2 & 0 & 0 & 2 & -1 & 1 & -1 & 2 & -2 & -3 & 2 & -1 & 0 & 0 & 0 & 0 & -1 \\
0 & -1 & 1 & 0 & 0 & 1 & 0 & 0 & 0 & 1 & -1 & -2 & 1 & -1 & 0 & 0 & 0 & 0 & 0 \\
1 & 0 & 0 & 0 & 0 & 0 & 0 & 0 & 0 & 0 & 1 & 1 & -1 & 1 & 1 & 0 & 0 & 0 & 0 \\
1 & 1 & 0 & 0 & 0 & -1 & 0 & 0 & 0 & -1 & 2 & 2 & -1 & 1 & 0 & 0 & 0 & 0 & 1 \\
0 & 1 & -1 & 0 & 0 & -1 & 1 & -1 & 1 & 0 & 0 & 1 & 0 & 0 & 0 & 0 & 0 & 0 & 1 \\
0 & 1 & -1 & 0 & 0 & -1 & 1 & -1 & 1 & -1 & 1 & 2 & 0 & 0 & 0 & 0 & 0 & 0 & 1 \\
0 & -1 & 1 & 0 & 0 & 1 & -1 & 0 & 0 & 1 & -1 & -1 & 1 & -1 & 0 & 0 & 0 & 0 & 0 \\
1 & 2 & -1 & 0 & 0 & -2 & 1 & 0 & 0 & -1 & 2 & 2 & -1 & 1 & 0 & 1 & 1 & 0 & 1 \\
1 & 0 & 0 & 0 & 0 & 0 & 0 & 1 & -1 & 0 & 1 & 0 & 0 & 0 & 0 & 1 & 0 & 0 & 0 \\
1 & 1 & -1 & 0 & 1 & -1 & 0 & 0 & 0 & -1 & 2 & 2 & -1 & 1 & 0 & 1 & 0 & 0 & 0 \\
1 & -1 & 1 & 1 & 0 & 0 & -1 & 1 & -1 & 0 & 1 & 0 & 0 & 0 & 0 & 1 & 0 & 0 & 0 \\
-1 & -2 & 1 & 0 & 0 & 2 & -1 & 0 & 0 & 1 & -2 & -2 & 2 & -1 & 0 & -1 & 0 & 0 & -1 \\
0 & 2 & -1 & 0 & 0 & -2 & 1 & -1 & 0 & -2 & 2 & 3 & -1 & 1 & 0 & 0 & 0 & 0 & 1 \\
0 & -2 & 1 & 0 & 0 & 2 & -1 & 1 & -1 & 1 & -1 & -2 & 1 & 0 & 0 & 0 & 0 & 0 & -2
\end{array}\right)
$$

Let us define

$$
S=\sum_{j=1}^{9} A_{i}, S^{\prime}=\sum_{j=1}^{9} A_{i}^{\prime}
$$

The classes of the $(-2)$-curves $\left(R_{i}\right)$ defined in Section 4.3 are

$$
\left(R_{i}\right)=2 L-\frac{1}{3}\left(S+2 S^{\prime}+3 A_{i}+3 A_{i}^{\prime}\right),
$$

where $L$ is the pullback of a line by the double cover map $X_{\lambda} \rightarrow \mathbb{P}^{2}$. The translation automorphism $\tau$ defined in Section 4.3 sends $L$ to the class

$$
L^{\prime}=7 L-\frac{4}{3}\left(S+2 S^{\prime}\right)
$$


The three polynomials $A, B, D$ in $\mathbb{Q}(\omega)(t)$ of Section 4.4 are defined as follows:

$$
\begin{aligned}
& A=\left(\lambda^{3} t^{2}+3 \lambda^{3}-4 t^{2}\right)\left(\lambda^{3} t^{2}+3 \lambda^{3}+(6 \omega+6) \lambda^{2} t^{2}+(-6 \omega-6) \lambda^{2}-4 t^{2}\right) \\
& \quad \cdot\left(\lambda^{3} t^{2}+3 \lambda^{3}-6 \lambda^{2} t^{2}+6 \lambda^{2}-4 t^{2}\right)\left(\lambda^{3} t^{2}+3 \lambda^{3}-6 \omega \lambda^{2} t^{2}+6 \omega \lambda^{2}-4 t^{2}\right), \\
& B=\left(\lambda^{6} t^{4}+6 \lambda^{6} t^{2}+9 \lambda^{6}+6 \lambda^{5} t^{4}+12 \lambda^{5} t^{2}-18 \lambda^{5}-18 \lambda^{4} t^{4}+36 \lambda^{4} t^{2}-18 \lambda^{4}\right. \\
& \left.-8 \lambda^{3} t^{4}-24 \lambda^{3} t^{2}-24 \lambda^{2} t^{4}+24 \lambda^{2} t^{2}+16 t^{4}\right) \\
& \cdot\left(\lambda^{6} t^{4}+6 \lambda^{6} t^{2}+9 \lambda^{6}+(-6 \omega-6) \lambda^{5} t^{4}+(-12 \omega-12) \lambda^{5} t^{2}+(18 \omega+18) \lambda^{5}-18 \omega \lambda^{4} t^{4}\right. \\
& \left.+36 \omega \lambda^{4} t^{2}-18 \omega \lambda^{4}-8 \lambda^{3} t^{4}-24 \lambda^{3} t^{2}+(24 \omega+24) \lambda^{2} t^{4}+(-24 \omega-24) \lambda^{2} t^{2}+16 t^{4}\right) \\
& \cdot\left(\lambda^{6} t^{4}+6 \lambda^{6} t^{2}+9 \lambda^{6}+6 \omega \lambda^{5} t^{4}+12 \omega \lambda^{5} t^{2}-18 \omega \lambda^{5}+(18 \omega+18) \lambda^{4} t^{4}\right. \\
& \left.\quad+(-36 \omega-36) \lambda^{4} t^{2}+(18 \omega+18) \lambda^{4}-8 \lambda^{3} t^{4}-24 \lambda^{3} t^{2}-24 \omega \lambda^{2} t^{4}+24 \omega \lambda^{2} t^{2}+16 t^{4}\right), \\
& \quad D=((\lambda+2) t-(2 \omega+1) \lambda)((\lambda-2 \omega-2) t-(2 \omega+1) \lambda)((\lambda+2 \omega) t-(2 \omega+1) \lambda) \\
& \cdot\left(t^{2}-1\right)((\lambda+2) t+(2 \omega+1) \lambda)((\lambda-2 \omega-2) t+(2 \omega+1) \lambda)((\lambda+2 \omega) t+(2 \omega+1) \lambda) .
\end{aligned}
$$

\section{REFERENCES}

[1] Artebani M., Dolgachev I., The Hesse pencil of plane cubic curves, Ens. Math. (2) 55 (2009), no. 3-4, 235-273.

[2] Beauville A., Complex algebraic surfaces, 2nd ed. Lond. Math. Soc., 34. Cambridge University Press, 1996. $\mathrm{x}+132 \mathrm{pp}$.

[3] J. Bertin, Réseaux de Kummer et surfaces K3, Invent. Math. 93 (1988), no. 2, 267284.

[4] Barth W., K3 surfaces with nine cusps. Geom. Dedicata 72 (1998), no. 2, 171-178.

[5] Barth W., On the classification of K3 surfaces with nine cusps, Complex analysis and algebraic geometry, de Gruyter, Berlin, 2000, pp. 41-59. MR 1760871.

[6] Barth W., Hulek K., Peters C.A.M., Van de Ven A., Compact complex surfaces. Sd ed. Erg Math Grenz. 3. Folge., 4. Springer-Verlag, Berlin, 2004. xii+436 pp.

[7] Birkenhake C., Lange H., A family of Abelian surfaces and curves of genus four, Manus. Math. 85, (1994), 393-407.

[8] Bosma W,. Cannon J., Playoust C., The Magma algebra system. I. The user language, Computational algebra and number theory (London, 1993), J. Symbolic Comput. 24, 1997, 3-4, 235--265.

[9] Conway J. H., Sloane N. J. A., Sphere packings, lattices and groups, Sde. ed., Grund. der Math. Wiss 290. Springer-Verlag, New York, 1993. xliv+679 pp.

[10] Dolgachev I., Abstract configurations in algebraic geometry, The Fano Conference, 423-462, Univ. Torino, Turin, 2004.

[11] Dolgachev I., Laface A., Persson U., Urzúa G, Chilean configuration of conics, lines and points, preprint.

[12] Griffiths P., Harris J., Principles of Algebraic Geometry, Wiley-Interscience, New York, 1978. xii +813 pp.

[13] Gritsenko V., Hulek K., Minimal Siegel modular threefolds, Math. Proc. Cambridge Philos. Soc. 123 (1998), 461-485.

[14] Huybrecht D., Lectures on K3 surfaces, Camb. Stud. Adv. Math., 158, Cambridge University Press, 2016. xi +485 pp.

[15] Miranda R., The Basic Theory of Elliptic Surfaces, Dottorato di Ricerca in Matematica, ETS Editrice, Pisa, 1989. vi+108 pp.

[16] Morrison D. R., On K3 surfaces with large Picard number. Invent. Math. 75 (1984), no. $1,105-121$.

[17] Nikulin V.V., On Kummer surfaces, Izv. Akad. Nauk SSSR Ser. Mat. 39 (1975), 278-293. English translation: Math. USSR. Izv, 9 (1975), 261-275. 
[18] Nikulin V.V., Integer symmetric bilinear forms and some of their geometric applications, Izv. Akad. Nauk SSSR Ser. Mat., 43, 1979, no 1, 111-177.

[19] Pokora P., Szemberg T., Conic-line arrangements in the complex projective plane, preprint, ArXiv 2002.01760

[20] Roulleau X., On the geometry of K3 surfaces with finite automorphism group: the compact case, preprint, ArXiv 1909.01909

[21] Roulleau X., Sarti A., Construction of Nikulin configurations on some Kummer surfaces and applications, Math. Annalen 373 (2019), no 11, 7651-7668.

[22] Roulleau X., Sarti A., Explicit Nikulin configurations on Kummer surfaces, preprint, ArXiv 1907.12215

[23] Sarti A., Transcendental lattices of some K3-surfaces, Math. Nachr. 281 (2008), no. 7, 1031-1046.

[24] Saint Donat B., Projective models of K-3 surfaces. Amer. J. Math. 96 (1974), 602-639.

[25] Schütt M., Shioda T., Mordell-Weil lattices, Erg. der Math. 3. Folge. A Series of Modern Surveys in Mathematics 70. Springer, 2019. xvi+431 pp.

[26] Silverman J., Advanced topics in the arithmetic of elliptic curves, Graduate Texts in Mathematics, 151. Springer-Verlag, New York, 1994. xiv+525 pp.

[27] Shimada I., On elliptic K3 surfaces, Michigan Math. J. 47 (2000), no. 3, 423-446.

[28] Shimada I., On elliptic K3 surfaces, arXiv:math/0505140

[29] Shioda T., On elliptic modular surfaces. J. Math. Soc. Japan 24 (1972), 20-59.

[30] Shioda T., Some remarks on abelian varieties, J. Fac. Sci. Univ. Tokyo Sect. IA 24 (1977),11-21.

David Kohel, Xavier Roulleau,

Aix-Marseille Université, CNRS, Centrale Marseille,

I2M UMR 7373,

13453 Marseille, France

David.Kohel@univ-amu.fr

Xavier.Roulleau@univ-amu.fr

Alessandra Sarti,

Laboratoire de Mathématiques et Applications, UMR CNRS 7348,

Université de Poitiers, Téléport 2,

Boulevard Marie et Pierre Curie,

86962 Futuroscope Chasseneuil, France

sarti@math.univ-poitiers.fr

http://www-math.sp2mi.univ-poitiers.fr/ sarti/ 